\title{
Plasticity of the human IgM repertoire in response to long-term spaceflight
}

Running title: Diversity of cosmonaut IgM repertoire

Judith-Irina Buchheim ${ }^{1 *}$, Stéphanie Ghislin ${ }^{2 *}$, Nassima Ouzren ${ }^{2}$, Eliane Albuisson ${ }^{3}$, Anne Vanet ${ }^{4,5}$, Sandra Matzel $^{1}$, Sergey Ponomarev ${ }^{6}$, Marina Rykova $^{6}$, Alexander Choukér ${ }^{1 \$}$ and Jean-Pol Frippiat ${ }^{2 \$ \#}$

${ }^{1}$ Laboratory of Translational Research "Stress and Immunity", Department of Anesthesiology, Hospital of the Ludwig-Maximilians-University, Munich, Germany.

${ }^{2}$ Stress Immunity Pathogens Laboratory, EA7300, Faculty of Medicine, Lorraine University, Vandoeuvrelès-Nancy, France.

${ }^{3}$ Nancy University Hospital, DRCI, MPI department, Methodology unit, Data management and Statistics UMDS, Vandoeuvre-lès-Nancy, France.

${ }^{4}$ University of Paris, Paris, France.

${ }^{5}$ Genoinformatics center, Jacques Monod Institute, UMR7592, CNRS, Paris, France.

${ }^{6}$ Institute of Biomedical Problems, Russian Academy of Sciences, Moscow, Russia.

* J.-I. Buchheim and S. Ghislin should be considered joint first author.

\$ A. Choukér and J.-P. Frippiat should be considered joint senior author.

\#Correspondence: Prof. J.-P. Frippiat, Stress Immunity Pathogens Laboratory, EA 7300, Faculty of Medicine, Université de Lorraine. 9 Avenue de la Forêt de Haye. F-54500 Vandœuvre-lès-Nancy, France. E-mail: jean-pol.frippiat@univ-lorraine.fr. Telephone: + 33372746286. 


\section{ABBREVIATIONS}

ANOVA, analysis of variance; B2M, beta-2-microglobulin; BLAST, Basic Local Alignment Search Tool; CDR3, third complementarity-determining region; CST, current stress test; EVA, extravehicular activities; $\mathrm{Geq} / \mathrm{ml}$, genome equivalents par ml; GHz, gigahertz; HTS, high-throughput sequencing; HSV, herpes simplex virus; IGHD, immunoglobulin heavy chain diversity gene segment; IGHJ, immunoglobulin heavy chain joining gene segment; IGHV, immunoglobulin heavy chain variable gene segment; IGKJ, immunoglobulin kappa light chain joining gene segment; IGKV, immunoglobulin kappa light chain variable gene segment; IGLJ, immunoglobulin lambda light chain joining gene segment; IGLV, immunoglobulin lambda light chain variable gene segment; IQR, interquartile range; ISS, International Space Station; LME, linear mixed effects model; M-MLV, Moloney Murine Leukemia Virus; RAG, recombination-activating gene; RSS, recombination signal sequences; RT-qPCR, reverse transcription quantitative polymerase chain reaction; TdT, terminal deoxynucleotidyl transferase; UBC, ubiquitin C.

\section{ACKNOWLEDGMENTS}

We thank all operators, scientists and administrators at Roscosmos (the Russian Space Agency), IBMP (the Institute for Biomedical Problems) and TsNIIMash in Russia, at DLR (the German Space Agency), at ESA (the European Space Agency) Moscow Office, at CNES (the French Space Agency) (Didier Chaput) as well as the team from the NASA Kennedy Space Centre and the Johnson Space Centre, who all made this project possible. We also express our thanks to Dr. Dominique Moser, Prof. Dr. Matthias Feuerecker, Marion Hörl and Katharina Biere for their support and to the former staff of the Thiel/Choukér laboratory, Prof. Dr. Manfred Thiel, PD Dr. Ines Kaufmann, Andrea Boltendahl, Camilla Ladinig and Iva Kumprey for their helping hands in realizing this project. We like to thank very much Dr. Bernd Johannes at DLR for support with the LME model and Dr. Andreas Osterman at the Max von Pettenkofer-Institute of the LMU, Munich, Germany, for the quantification of HSV-1/2 DNA. We also express our thanks to the ISS crew members who participated in this study with outstanding professionalism and enthusiasm.

This work was supported by CNES (grants DAR 4800000894, 4800000950 and 4800001008), the French Ministry of Higher Education and Research, the Université de Lorraine, the French State-Region Project Contract (CPER), the DLR on behalf of the Federal Ministry of Economics and Technology/Energy (grants BMWi 50WB0719, 50WB0919 and 50WB1319), ESA (ELIPS 3 and 4 programs), Roscosmos and the program of fundamental research (theme 65.1) of IBMP, Moscow.

In honor and memory of the cosmonaut, vice director of the IBMP and IMMUNO-co-PI Prof. Boris Morukov who passed away in 2015.

\section{CONFLICTS OF INTEREST STATEMENT}

The authors declare that they have no conflict of interest. 


\title{
AUTHOR CONTRIBUTIONS
}

A. Choukér and J.-P. Frippiat designed research; S. Ghislin, J.-I. Buchheim, N. Ouzren-Zarhloul and S. Matzel performed research; J.-I. Buchheim, S. Ghislin and E. Albuisson performed statistical analyses; A. Vanet conceived the software required for this project; S. Ghislin and J.-I. Buchheim analyzed data; S. Ghislin, J.-I. Buchheim, A. Choukér and J.-P. Frippiat wrote the manuscript in conjunction with S. Ponomarev and M. Rykova.

\begin{abstract}
Immune dysregulation is among the main adverse outcomes of spaceflight. Despite the crucial role of the antibody repertoire in host protection, the effects of spaceflight on the human antibody repertoire are unknown. Consequently, using high-throughput sequencing, we examined the IgM repertoire of five cosmonauts 25 days before launch, after $64 \pm 11$ and $129 \pm 20$ days spent on the International Space Station (ISS), and at 1, 7 and 30 days after landing. This is the first study of this kind in humans. Our data revealed that the $\operatorname{IgM}$ repertoire of the cosmonauts was different from that of control subjects $(n=4)$ prior to launch and that two out the five analyzed cosmonauts presented significant changes in their IgM repertoire during the mission. These modifications persisted up to 30 days after landing, likely affected the specificities of IgM binding sites, correlated with changes in the $\mathrm{V}(\mathrm{D}) \mathrm{J}$ recombination process responsible for creating antibody genes, and coincided with a higher stress response. These data confirm that the immune system of approximately half of the astronauts who spent six months on the ISS is sensitive to spaceflight conditions, and reveal individual responses indicating that personalized approaches should be implemented during future deep-space exploration missions that will be of unprecedented durations.
\end{abstract}

KEYWORDS: antibody, cosmonaut, spaceflight, stress, V(D)J recombination 


\section{MAIN TEXT}

\section{INTRODUCTION}

Spaceflight-associated immune system dysregulation has long been acknowledged. Fifteen out of 29 Apollo astronauts developed bacterial or viral infections during, immediately after, or in the week after landing. ${ }^{1}$ Recent work analyzing medical data collected from 46 astronauts who spent six months onboard the International Space Station (ISS) corroborated these initial findings. ${ }^{2}$ This study revealed that $46 \%$ of them faced immunological problems, with infection being the second most frequent event.

During spaceflight, the crew experiences a multitude of unique stressors, such as microgravity, radiation, isolation, confinement, disrupted sleep and circadian rhythms, high expectations in terms of performance, and the risk of equipment failure or fatal mishaps. The combination of these stressors constitutes the space "exposome", which exerts its altering effects on various organ systems, including the immune system. ${ }^{3}$ In addition to shifts in peripheral leukocyte distribution and function, altered cytokine levels are observed that positively correlate with latent virus reactivation in individual crewmembers. ${ }^{4}$ Despite the fact that immune alterations, together with limited therapeutic options, represent a serious risk to crewmembers during future long-term space missions such as the deployment of the Lunar Gateway and possible interplanetary missions, ${ }^{3,5-8}$ limited data are available on the antibody repertoire of astronauts. To date, most studies have quantified the total levels of circulating antibody isotypes, but these studies have produced conflicting results and have not considered the antibody binding site diversity required to ensure efficient host protection. ${ }^{9-14}$ However, some studies have suggested that humoral immunity might be affected during a long-term mission even if spaceflight does not seem to have a major impact on the numbers and proportions of circulating B-cell subsets in crewmembers staying 6 months onboard the ISS. ${ }^{13}$ Indeed, it was shown that a 5-month spaceflight affects amphibian antibody production in response to antigen stimulation, ${ }^{15-17}$ and reduces the frequency of somatic hypermutation that diversifies antibody binding sites to improve their affinity. ${ }^{18}$ These observations are supported by a study demonstrating that hindlimb unloading, a ground-based model frequently used to simulate microgravity, ${ }^{19}$ significantly reduces the number of murine pro-B cells and strongly reduces the number of pre-B cells, two maturation stages at which antibody gene assembly occurs in bone marrow. ${ }^{20}$ Furthermore, two studies revealed some changes in antibody gene segment usage in mice subjected to 4 weeks of hindlimb unloading. ${ }^{21,22}$ Finally, it is interesting to note that reduced B lymphopoiesis was also detected in mice that spent 30 days onboard the BION-M1 biosatellite, ${ }^{23}$ thereby suggesting that extended exposure to spaceflight conditions could modify the diversity of the antibody repertoire.

This diversity, which is essential for host protection, results from the random association of antibody gene segments: a variable (IGKV or IGLV) and a joining (IGKJ or IGLJ) gene segment to generate a kappa or a lambda light chain gene as well as a variable (IGHV), a diversity (IGHD) and a joining (IGHJ) gene segment to produce a heavy chain gene. These associations are created during B lymphopoiesis within the 
bone marrow thanks to the $\mathrm{V}(\mathrm{D}) \mathrm{J}$ recombination process. $\mathrm{V}(\mathrm{D}) \mathrm{J}$ recombination requires recombinationactivating gene (RAG) proteins that interact with recombination signal sequences (RSS) located at the 3' end of IGHV, on both sides of IGHD and at the 5' end of IGHJ segments, and inflict DNA breaks between coding IGHV, IGHD or IGHJ segments and noncoding RSS, leading to the formation of hairpin-sealed coding ends. ${ }^{24}$ In further steps, the Artemis protein complex opens hairpin loops, eventually creating palindromic sequences corresponding to short inverted repeats of the segment terminal sequence. ${ }^{25}$ This is followed by nucleotide addition through terminal deoxynucleotidyl transferase (TdT), hybridization of single stranded DNA on a few nucleotides, and ligation. The end-product is the third complementaritydetermining region (CDR3) of Ig chains, a short and highly variable region responsible for much of the diversity of antigen binding sites.

Given the absence of information about the effect of extended spaceflight conditions on the human antibody repertoire, we studied the repertoire of $\operatorname{IgM}$ heavy chains from cosmonauts participating in longterm missions on the ISS and evaluated their stress level as well as the activities of RAG effectors required for $\mathrm{V}(\mathrm{D}) \mathrm{J}$ recombination. We focused on the $\operatorname{IgM}$ repertoire since $\operatorname{IgM}$ is one of the antigen receptors of naïve mature B-cells and is the first antibody isotype produced following an encounter with an antigen. Overall, our data show that cosmonauts respond individually to the spaceflight environment, affecting their personal IgM repertoire diversity, $\mathrm{V}(\mathrm{D}) \mathrm{J}$ recombination, and stress response.

\section{MATERIALS AND METHODS}

\subsection{Study design}

This longitudinal study was carried out in accordance with the recommendations from the ethical standards of the appropriate institutional and national committees and with the World Medical Association Helsinki Declaration of 1975 (revised in 2008). The protocol (study name: IMMUNO) for cosmonauts was evaluated by the Russian Ethical Board (Biomedicine Ethics Committee) at the Institute of Biomedical Problems (IBMP), Moscow, and was awarded the protocol number MBI-29. The protocol was also approved by the ESA Medical Board and the Russian Space Agency (Roscosmos). The local Ethical Committee of Ludwig-Maximilian University (LMU), Munich, approved the protocol for the control group (protocol numbers 025/02 and 269-15). All subjects gave written informed consent after being informed of the aim and objectives of the project. Blood and saliva were collected from 5 male cosmonauts with a median age of $48 \pm 2$ (IQR, interquartile range) years (ranging from 39-54 years) who were assigned to a long-term spaceflight mission (ranging from 124 to 186 days) on the ISS. Two of these cosmonauts (COS2 and COS3) took part in the same expedition. Blood and saliva collection occurred at 6 time points. Sample collection on the ground was performed at the Gagarin Cosmonaut Training Center near Moscow or at the DLR training facility in Cologne, Germany, or at the Johnson Space Center (JSC) at NASA, Houston, Texas. Baseline data collection occurred 25 days before flight $(\mathrm{L}-25, \mathrm{n}=5)$. Inflight samplings occurred after $64 \pm 11(\mathrm{~F}+64$, 
$\mathrm{n}=4)$ and $129 \pm 20(\mathrm{~F}+129, \mathrm{n}=3)$ days spent on the ISS (indicated values are means \pm the standard deviation). Postflight sampling occurred on days $1(R+1, n=5), 7(R+7, n=5)$ and $30(R+30, n=3)$ after landing. A control group of 4 males with a median age of $41 \pm 4$ (IQR) years (ranging from 36 to 46 years) was selected according to the following criteria: (1) adult, (2) male, (3) age > 35 years, (4) no systemic pathology and (5) not receiving any daily medication. Blood and saliva were collected from these volunteers at T0 and T+8 months. On-ground-collected blood samples were either used directly for B-cell quantification or immediately stored at $-80^{\circ} \mathrm{C}$, as saliva samples, until further analysis. Onboard the ISS, blood and saliva samples were stored frozen inside the MELFI freezer at $-100^{\circ} \mathrm{C}$. Frozen samples return to Earth and to the investigators' laboratories was performed according to standard ESA protocols.

\subsection{Total B-cell quantification}

Relative counts $(\%)$ of B-lymphocytes $\left(\mathrm{CD} 19^{+}\right)$were determined before the flight and after landing from $1 \mathrm{ml}$ of lithium heparinized fresh whole blood using the BD Multitest ${ }^{\mathrm{TM}}$ IMK kit (BD Biosciences, Heidelberg, Germany) according to the manufacturer's instructions.

\subsection{RNA extraction and RT-qPCR}

Total RNA was extracted from $300 \mu$ l of whole blood using the Nucleospin ${ }^{\circledR}$ RNA blood kit (Macherey Nagel, Hoerdt, France) and reverse transcribed using random primers, RNaseOUT and M-MLV reverse transcriptase following the manufacturer's instructions (Invitrogen, Cergy Pontoise, France). qPCR was conducted using a Mastercycler ${ }^{\circledR}$ realplex $^{2}$ real-time PCR machine (Eppendorf, Hamburg, Germany). Each qPCR assay was performed in triplicate using Takyon No ROX SYBR 2X MasterMix blue dTTP (Eurogentec, Seraing, Belgium). The cycling protocol was as follows: $3 \mathrm{~min}$ at $95^{\circ} \mathrm{C}$ followed by 40 cycles of $15 \mathrm{~s}$ at $95^{\circ} \mathrm{C}$ and $30 \mathrm{~s}$ at the annealing temperature indicated in Table 1. IgM and $\operatorname{IgD}$ heavy chain mRNA relative expression levels were normalized to two housekeeping transcripts (ubiquitin C (UBC) and beta-2microglobulin (B2M)) as previously described. ${ }^{26}$ The stability of housekeeping transcripts was checked using BestKeeper software. ${ }^{27}$ Primer pairs were designed in different exons to ensure that they could not hybridize to potential traces of genomic DNA, and their specificity was checked using a BLAST search through the U.S. National Center for Biotechnology Information database (Bethesda, MD, USA).

\subsection{Stress questionnaire}

The current stress test (CST) is a validated and standardized one-page paper-based self-evaluation that is completed in $<1 \mathrm{~min}$ and is therefore easily applicable to the present study. ${ }^{28}$ The CST aims to determine an individual's psychological state repeatedly under the conditions of acute and chronic stress. Because of the composition of the questionnaire, there is no carryover effect, which is a prerequisite for the application of the test in longitudinal studies. In this test, the subject must decide between six pairs of 
contradictory feelings of increasing intensities. From the sum of the obtained values, the final CST score is calculated in the range from 1 (no stress) to 6 (maximal stress).

\subsection{Salivary cortisol quantification}

Saliva samples were collected in the morning (7:00-8:00 AM) and in the evening (7:00-8:00 PM) before food ingestion or dental hygiene measures. Saliva was collected from subjects by having them chew on a sterile cotton swab for 30-45 s (Salivette ${ }^{\circledR}$, Sarstedt, Germany). On ground and inflight samples were frozen and stored until analysis. Free cortisol levels were quantified with an automated immunoassay system based on the principle of electrochemiluminescence (Elecsys 2010, Roche, Mannheim, Germany) at the Institute of Clinical Chemistry, Hospital of the University of Munich, Germany.

\subsection{Quantification of HSV DNA in saliva}

Quantification of HSV-1/2 DNA was performed using the accredited routine diagnostics laboratory of the Max von Pettenkofer-Institute of the LMU, Munich, Germany. HSV-1/2 DNA was extracted from $200 \mu \mathrm{l}$ of saliva using a QIAsymphony instrument and reagents (QIAsymphony DSP Virus/Pathogen Mini Kit (QIAGEN GmbH, Hilden, Germany)). Total elution volume was $90 \mu 1$. Specific HSV-1/2 sequences were amplified from $5 \mu$ of extracted DNA using an Applied Biosystems ${ }^{\text {TM }} 7500$ Real-Time PCR System and Applied Biosystems ${ }^{\mathrm{TM}}$ TaqMan ${ }^{\mathrm{TM}}$ Universal PCR Master Mix (both Thermo Fisher Scientific Inc., Waltham, MA, USA) according to the manufacturer's instructions. Optimized primers and a fluorescein amidite (FAM)-labelled probe (both Ella Biotech GmbH Martinsried, Germany) amplified an 81-nucleotide sequence of the polymerase-gene in a reaction volume of $20 \mu 1$. Thermocycler settings were $50^{\circ} \mathrm{C}$ for $2 \mathrm{~min}$ and $95^{\circ} \mathrm{C}$ for $10 \mathrm{~min}$ followed by 45 cycles of $95^{\circ} \mathrm{C}$ for $15 \mathrm{~s}$ and $60{ }^{\circ} \mathrm{C}$ for $60 \mathrm{~s}$. Quantification was achieved by simultaneous amplification of four HSV-plasmid-DNA standards of $5 \times 10^{2}, 5 \times 10^{3}, 5 \times 10^{4}$ and $5 \times 10^{5}$ copies/ml. The lower limit of detection (LoD95) of the PCR system was $700 \mathrm{Geq} / \mathrm{ml}$.

\subsection{Illumina sequencing}

Whole blood RNA was reverse transcribed using random primers, RNaseOUT and MML-V reverse transcriptase (Invitrogen, Cergy Pontoise, France) following the instructions of the LymphoTrack ${ }^{\circledR}$ IGH Somatic Hypermutation MiSeq kit (InvivoScribe, San Diego, CA, USA). Then, $2.5 \mu 1$ of cDNA prepared from each blood sample was mixed with $22.5 \mu$ of IGH master mix provided in the kit (this master mix contains primers, with Illumina adapters, annealing to the leader and joining regions of IgM cDNAs thereby allowing the amplification of IgM cDNA 5'-ends) and $0.1 \mu 1$ of Taq Gold DNA polymerase (Invitrogen, Cergy Pontoise, France). These mixtures were subjected to the following cycling protocol: 7 min at $95^{\circ} \mathrm{C}$ followed by 35 cycles of $45 \mathrm{~s}$ at $95^{\circ} \mathrm{C}, 45 \mathrm{~s}$ at $60^{\circ} \mathrm{C}, 1 \mathrm{~min} 30 \mathrm{~s}$ at $72^{\circ} \mathrm{C}$ and finally $10 \mathrm{~min}$ at $72^{\circ} \mathrm{C}$. The quantities and sizes of the amplification products were validated using the Agilent High Sensitivity DNA 
assay (Agilent, Santa Clara, USA). High-throughput sequencing of libraries was conducted using the MiSeq Illumina apparatus of UMS2008 from Lorraine University (Vandoeuvre-lès-Nancy, France) using Illumina's standard protocol. Sequences were first treated using the LymphoTrack ${ }^{\circledR}$ IGH SHM MiSeq software. Then, complete sequences were analyzed using IMGT/High V-quest software (http://www.imgt.org). ${ }^{29}$ Finally, the obtained data were analyzed using a specific software (see below). Sequencing data have been deposited to the NCBI Sequence Read Archive (https://www.ncbi.nlm.nih.gov/sra) under accession number PRJNA627324.

\subsection{Software dedicated to the analysis of sequences}

A software written in Perl, an efficient editing language, was created to calculate the parameters necessary for this study. This software parsed twenty-eight different files corresponding to twenty-eight different blood samples. One to two seconds of execution was necessary to analyze a file using a $2.9 \mathrm{GHz}$ Intel Core i9 processor. This execution time was proportional to the number of lines to process, with each line corresponding to one sequence. On average, there were 233819 lines per sample. The software examined each row and defined those having a CDR3 sequence and productive functionality. A sequence was considered productive if it was in frame and did not contain a premature stop codon. The redundancy of this set of CDR3 sequences was then calculated. A new set of results consisting of one representative of each CDR3 sequence expressed one or more times was then generated. The mean number of sequences with unique CDR3 was 41072 per sample (Table 2). From this set of results, the following parameters were calculated: $i$ ) the usage frequency of each IGHV, IGHD and IGHJ segment; $i i$ ) the frequency of IGHV replacement footprints as defined by Lange et al. $;^{30}$ iii) the number of nucleotides deleted on both sides of IGHD segments as well as at the 3'-IGHV and 5'-IGHJ extremities; and $i v$ ) associations of IGHV segments of the IGHV3 and IGHV4 subgroups with IGHJ4 to IGHJ6 segments.

\subsection{Analysis of CDR3 amino acid sequences}

The number of each amino acid at each position within CDR3 regions ranging from 9 to 24 AA was determined using Weblogo3 (http://weblogo.threeplusone.com/create.cgi). ${ }^{31}$ Then, dispersion indexes considering both the position and the frequency of each amino acid along CDR3 sequences were calculated as described below.

\subsection{Statistics}

\section{Calculation of dispersion indexes}

Dispersion indexes were used to determine whether differences in the repertoire of VDJ associations, or other criteria, exist between groups or time points. ${ }^{32}$ The following formula shows, for example, how these indexes (comprised between 0 and 1), were calculated for VDJ associations. These dispersion indexes take into account all associations whatever their frequencies of expression. 
$\mathrm{I}($ treated - reference $)=0.5^{*} \sum \mid \mathrm{F}$ of $\mathrm{VDJ}_{\mathrm{i}}$ from treated $-\mathrm{F}$ of $\mathrm{VDJ}_{\mathrm{i}}$ from reference $) \mid$

$$
\mathrm{i}=1
$$

where ' $F$ ' is the frequency of a VDJ association in one group or at one time point,

' $\mathrm{n}$ ' is the number of different VDJ associations detected in our NGS data,

'treated' corresponds to values determined for cosmonauts during or after the flight or to values determined for control subjects at T+ 8 months depending on the comparison we wish to do, and

'reference' is the mean value determined for cosmonauts before launch or for control subjects at T0.

Given the low number of subjects, the means and confidence intervals of dispersion indexes were determined for control subjects and used as references. Cosmonaut dispersion indexes were compared to these intervals, with those being outside control confidence intervals were considered different. These comparisons were used to construct heat maps in which values within confidence intervals are indicated in light gray, those above are in red and those below are in green, with color intensity reflecting the importance of the difference by comparison to the corresponding border.

\section{Statistical analysis of data}

Statistical evaluation of the data set was performed using SPSS 25 (IBM, USA). Outcome variables and their residuals were tested for normal distribution by applying the Kolmogorov-Smirnov test. Box-Cox transformation of data was performed where indicated and only when residuals of the tested variable were found not to be normally distributed. Data analysis within a single group was performed employing a linear mixed effects (LME) model, where the time points measured were regarded as fixed and subject as random effects. For cosmonaut subgroup comparisons, the LME was used where subgroup, time points and the interaction of subgroup and time point were considered fixed effects. Subjects were considered random effects. A Bonferroni correction was employed as a post hoc test. Simple comparisons between two groups were performed using an unpaired T-test for normally distributed data and a Mann-Whitney U-test for nonnormally distributed data, where indicated. A value of $\mathrm{p}<0.05$ was considered statistically significant.

For multiple comparisons (Table 2), the normality of the distribution was determined using the Shapiro-Wilk normality test, and homogeneity of variance was confirmed using the Levene test. As the normality of the distribution and homogeneity of variance were validated, one-way ANOVA was performed.

For correlations, distribution normality was assessed using the Shapiro-Wilk normality test, and then correlation was tested using the unilateral ( $r>0$ ) Bravais-Pearson correlation test. A unilateral test was used, as we hypothesized that dispersion indexes for the cosmonaut IgM repertoire and RAG signatures, reflecting RAG activity, would evolve in the same direction. A value of $\mathrm{p}<0.05$ was considered statistically significant, and a $\mathrm{p}$ value between 0.05 and 0.10 was considered a tendency. 


\section{RESULTS}

\subsection{B-cells and IgM/IgD mRNAs in peripheral blood}

Before analyzing the cosmonauts' IgM repertoire 25 days before the flight (L-25), after $64 \pm 11$ $(\mathrm{F}+64)$ and $129 \pm 20(\mathrm{~F}+129)$ days spent on the ISS, and $1(\mathrm{R}+1), 7(\mathrm{R}+7)$ and $30(\mathrm{R}+30)$ days after landing, we determined the percentage of $\mathrm{CD} 19^{+} \mathrm{B}$-cells in the lymphocyte compartment of the peripheral blood using pre- and postflight cosmonaut as well as control group blood samples (it was not possible to perform such studies with inflight blood samples) (Figure 1). Whereas the percentage of B-cells remained very stable over 8 months in the control group (T0: $14.48 \pm 5.96 \%$ vs. $\mathrm{T}+8 \mathrm{~m}: 13.23 \pm 4.92 \%, \mathrm{p}=0.738$ ), a significant increase in B-cell percentages in cosmonauts directly after landing $(\mathrm{R}+1)$ compared to preflight values (L-25: $11.58 \pm 6.54 \%$ vs. $\mathrm{R}+1: 16.11 \pm 6.88 \%, \mathrm{p}=0.025)$ was quantified. This increase was transient as it returned to the baseline level after seven days on Earth. Additionally, IgM and IgD heavy chain mRNAs were quantified in pre-, in- and postflight as well as in control frozen blood samples. Figure 2 shows that relative expression levels of $\operatorname{IgM}$ and $\operatorname{IgD}$ heavy chain transcripts in peripheral blood were stable in control subjects, during the 6-month stay on the ISS and were not statistically increased at $\mathrm{R}+1$, indicating that spaceflight would not affect our ability to assess the IgM repertoire.

\subsection{IgM repertoire analysis}

To determine how spaceflight conditions affect the diversity of the IgM repertoire, we constructed IgM cDNA libraries, which were subjected to high-throughput sequencing (HTS), using RNA extracted from the blood of control subjects at two time points (T0 and T+8m) and of cosmonauts at six time points ( $\mathrm{L}-25, \mathrm{~F}+64, \mathrm{~F}+129, \mathrm{R}+1, \mathrm{R}+7$ and $\mathrm{R}+30)$. Unique sequences were extracted from HTS data to eliminate potential PCR biases and clonal expansions. This selection was based on CDR3 sequences because it is the most diverse part of IgM transcripts. Table 2 presents the number of obtained unique sequences in controls and cosmonauts. Eleven to fifteen percent of these sequences were nonfunctional (Figure 3A). This percentage of nonfunctional sequences was stable in controls and cosmonauts over time (p values always $>0.05$ when comparing percentages of unproductive sequences in controls and cosmonauts, regardless of the considered time point). These nonfunctional sequences were not further considered, as the purpose of this study was to determine the impact of spaceflight on IgM diversity. We also assessed the efficiency of the kit used to create our libraries by determining which functional IGHV gene segments are found in our datasets issued from the 5 cosmonauts and the 4 controls. According to the IMGT database, the genomic IGH repertoire encoded by the human IGH locus comprises 38-46 functional IGHV gene segments depending on the haplotypes. Table 3 shows that 42-43 functional IGHV gene segments were detected in our datasets. We are therefore confident that our libraries provide an accurate picture of the IGH rearrangements. This is supported by previous studies having shown that detected IGHV gene segments assessed without amplification parallel the repertoires reported using more focused amplification methods. ${ }^{33,34}$ 
Then, we wondered whether the usages of IGHV and IGHD subgroups and of IGHJ segments were affected. The analysis of their expression in our libraries revealed that this was not the case (Figure 3B). The same biases and expression frequencies were noted over time in controls and cosmonauts.

To precise this first global analysis, we studied associations between IGHV, IGHD and IGHJ gene segments in control subjects and in cosmonauts over time, and dispersion indexes were calculated to determine whether differences in the repertoire of VDJ associations exist. We first compared indexes determined for controls at T0 with those of cosmonauts before launch (L-25) and those of controls after 8 months with those of cosmonauts 7 days after landing $(\mathrm{R}+7)$ (Figure 4A). These comparisons revealed that dispersion indexes of control subjects were stable over time, thereby showing the stability of their repertoire of VDJ associations in IgM heavy chains. Consequently, means of indexes determined for controls at T0 and $\mathrm{T}+8 \mathrm{~m}$ were used to define a reference confidence interval. However, statistically significant differences between control subjects and cosmonauts were observed at both time points, demonstrating that the cosmonaut IgM repertoire was already different from that of controls before the flight and that it was also different after landing. This difference in repertoire was corroborated by the analysis of footprints resulting from IGHV segment replacement (Figure 4B). IGHV replacement refers to RAG-mediated secondary recombination of previously rearranged IGHV segments. This process renews almost the entire V coding region but retains a short stretch of nucleotides as a replacement footprint at the newly generated IGHVIGHD junction. It allows rescuing B-cells having generated, for example, nonfunctional VDJ associations following $\mathrm{V}(\mathrm{D}) \mathrm{J}$ recombination and is involved in the generation of the primary B-cell repertoire during B lymphopoiesis. ${ }^{35}$

To determine the effects of spaceflight on the IgM repertoire, dispersion indexes calculated for VDJ associations in cosmonaut IgM repertoires analyzed using inflight and after-landing blood samples were compared to those determined 25 days before launch. We also compared dispersion indexes of controls at $\mathrm{T} 0$ and $\mathrm{T}+8 \mathrm{~m}$ to determine normal variation in VDJ associations (used as reference). Then, cosmonaut index variations were compared to normal variation determined using controls, and differences were expressed in a heat map. Figure 5A shows that two cosmonauts (COS4 and COS5) were consistently above the upper limit of the confidence interval determined using controls, suggesting that their VDJ associations were more variable than those of controls. The comparison of data between COS1, $2 \& 3$ versus COS $4 \& 5$, using the statistical LME model, presented a similar trend though the low number of individuals in each group must be kept in mind. The same analyses were then performed for VDJ associations involving IGHV segments of the most expressed IGHV subgroups (IGHV3 and IGHV4) and the most frequently used IGHJ segments (IGHJ4 to IGHJ6) (Figure 5B). These analyses confirmed that the IgM repertoire of COS4 and 5 was more modified than those of COS1, 2 and 3 during the stay in the ISS but also during the 30-day recovery period, as indicated by the abundance of orange rectangles at all considered time points indicating variations above the confidence interval determined using controls and rare green rectangles indicating less variations than in controls. Thus, the diversity of IgM heavy chains was affected in two out of the five studied cosmonauts. 
Furthermore, Figure 5B showed that some specific associations (IGHV3-48 with IGHJ5 and IGHV3-23 with IGHJ6) are altered in all the cosmonauts.

\subsection{Potential impact on immunity}

To evaluate the consequences of the modifications of the IgM repertoire in COS4 and COS5, we studied CDR3 regions. Indeed, heavy chain CDR3 is a major contributor to the antibody binding site because it encodes the longest and most variable loop composing this site. It is made of the 3 '-end of the IGHV, the entirety of the IGHD and the 5'-end of the IGHJ gene segments that are assembled during V(D)J recombination. We first analyzed the length of these regions and noted that this parameter is not impacted by spaceflight or during the recovery period (Figure 6A). The average length of IgM CDR3 was 15.7 amino acids (AA) in controls and cosmonauts, a value very close to the one (15.5 AA) determined in previous studies of IgM in healthy subjects. ${ }^{32,33}$ Then, we wondered whether CDR3 amino acid composition could be affected. We studied the position and the frequency of each amino acid along CDR3 sequences of 9 to 24 $\mathrm{AA}$ at the different time points. Dispersion indexes were calculated, and heat maps were constructed as explained above to reveal inflight and after-landing variations. Figure $6 \mathrm{~B}$ revealed more changes of CDR3 AA composition in COS4 and COS5 than in COS1, 2 and 3 during the flight and recovery periods. Thus, spaceflight modified CDR3 loops composing IgM binding sites in two out of the five studied cosmonauts suggesting that the specificities of $\operatorname{IgM}$ binding sites are likely affected in these two subjects.

\subsection{HSV reactivation}

Because previous work showed that viral reactivation is frequent during short and long-term space missions, ${ }^{4,36-40}$ we analyzed saliva samples for the presence of herpes simplex virus (HSV) type 1 and 2 DNA but did not detect HSV-1 or -2 DNA in any of the inflight samples.

\subsection{Impact on $\mathrm{V}(\mathrm{D}) \mathrm{J}$ recombination}

We also wondered if these IgM repertoire changes could result from a modification of the V(D)J recombination process. To address this question, we analyzed signatures left by RAG1/2 in IgM transcripts. Indeed, when these proteins cut DNA between coding segments and RSS during V(D)J recombination, some nucleotides (nt) can be deleted at the 3'-end of IGHV, on both sides of IGHD and at the 5'-end of IGHJ segments. Consequently, we studied IGHD length as well as nucleotides trimmed off of IGHV and IGHJ gene segments. These analyses were performed at the different time points, dispersion indexes were calculated for each signature, and heat maps were established to reveal inflight and after-landing differences by comparing these data to variations over time in control subjects. The results shown in Figure 7 indicate that COS4 and COS5 were systematically above the control confidence interval for 2 out of 3 signatures left by RAG (IGHD length and nt removed at 5'-IGHJ ends). The comparison of results between COS1, 2 \& 3 versus COS4 \& 5, using the statistical LME model, confirmed this difference, indicating that there was an 
inflight modification of the RAG1/2 activity in COS4 and COS5. To investigate the possible link between these activity changes and inflight modifications of the IgM repertoire, Bravais-Pearson correlation coefficients were determined. Interestingly, Table 4 shows that positive correlations between these parameters exist at the $\mathrm{F}+64$ and $\mathrm{R}+1$ time points and that a trend is still observed at $\mathrm{R}+7$. Correlations could not be evaluated at F+129 and $\mathrm{R}+30$ because the number of samples was too low.

\subsection{Analysis of stress parameters}

Since stress can modulate immunity, ${ }^{8}$ subjective and objective test methods were applied to evaluate stress in cosmonauts and controls in the morning and evening of a scheduled time point. Subjective stress levels were evaluated using the CST questionnaire. The mean CST scores were low overall, indicating a low personal stress level in both groups (Figure 8A). Furthermore, CST scores were stable in cosmonauts and controls over time ( $\mathrm{p}$ values always $>0.05$ when comparing scores in controls and cosmonauts regardless of the considered time point). Although the morning CST score in controls at the first time point was found to be significantly higher than that in the cosmonaut group (T0: $3.20 \pm 0.64 \mathrm{vs.} \mathrm{L-25:} 2.4 \pm 0.65, \mathrm{p}=0.04$ ), the absolute score was not in a range suggestive of stress. All other group comparisons were not statistically significant. Furthermore, we quantified cortisol in saliva samples. Circadian differences with higher levels in the morning and lower levels in the evening were observed in controls and cosmonauts throughout the mission, indicating a preserved circadian rhythm for cortisol. No significant changes were detected over time in controls for morning cortisol. Evening values showed a statistically significant increase over time, which was not considered physiologically relevant, since the values were still at a very low level (T0: $0.03 \pm$ $0.03 \mu \mathrm{g} / \mathrm{dl}$ vs. $\mathrm{T}+8 \mathrm{~m}: 0.05 \pm 0.02 \mu \mathrm{g} / \mathrm{dl}, \mathrm{p}=0.001)$. Cosmonauts did not show any significant changes over time for morning cortisol values. However, evening cortisol values exhibited a significant increase at F+64 during the mission and shortly after return (at $\mathrm{R}+1$ ), indicating a stress response. The significant increases observed can be attributed to COS4 and COS5, as the LME model revealed an increase in stress over time in these two subjects, which was not the case for the other three cosmonauts. Group comparisons showed no differences in morning cortisol levels, yet evening cortisol levels in cosmonauts were generally higher than control values before the mission (T0: $0.03 \pm 0.03 \mu \mathrm{g} / \mathrm{dl}$ vs. $\mathrm{L}-25: 0.13 \pm 0.06 \mu \mathrm{g} / \mathrm{dl}, \mathrm{p}=0.01$ ). It is worth noting that one cosmonaut (COS5) exhibited unusually high morning cortisol levels on F+64 for unknown reasons and high evening cortisol levels on $\mathrm{R}+1$, which was likely due to a ballistic return to Earth; i.e., the subject was exposed to $8 \mathrm{~g}$ during the landing phase, resulting in an unusual acute stress load even for a trained astronaut. 


\section{DISCUSSION}

In this study, we investigated whether a long-term space mission affects the repertoire of IgM heavy chains. To address this question, we analyzed the $\operatorname{IgM}$ transcriptome in the blood of cosmonauts and controls. To ensure that our transcriptomic data were not biased, we determined the percentage of blood $\mathrm{CD} 19^{+} \mathrm{B}$-cells before and after the flight, studied the relative expression of IgM and IgD heavy chain transcripts in the blood before, during and after the mission, and checked the efficiency of the kit used to create libraries of $\operatorname{IgM}$ transcripts. These analyses revealed an increase in B-cells at $\mathrm{R}+1$ which may perhaps be due to fluid redistributions that occur after landing. This increase does not correspond to an increase in transitional, mature naïve, non-switched or IgM-only memory B-cells, as no change in the relative expression of IgD and IgM was noted over time. ${ }^{41}$ In agreement with this conclusion, a previous study conducted on eight crewmembers involved in a 6-month mission to the ISS showed that spaceflight has no effect on the number and proportion of naïve, transitional and $\operatorname{IgM}^{+}$B-cell subsets. ${ }^{13}$ Nevertheless, in that study, no increase in total B-cells was noted on the landing day $(\mathrm{R}+0)$. This discrepancy could result from differences in crewmembers. However, the fact that the expression of IgM transcripts was not affected by a stay on the ISS ensures that potential IgM repertoire changes are not due to a modification of this parameter. Consistent with this conclusion, no statistically significant impacts on the numbers of unique IgM heavy chain mRNA sequences were observed, indicating that the flight did not affect our ability to assess the IgM repertoire and that there was no group bias in our transcriptome analysis.

We first observed that the IGHV1, IGHV3, IGHV4, IGHD1, IGHD2, IGHD3 and IGHD6 subgroups, as well as the IGHJ4, IGHJ5 and IGHJ6 segments, were detected at high levels in IgM heavy chain transcripts of controls and cosmonauts. These results are consistent with those of previous studies of the $\operatorname{IgM}$ repertoire of healthy adults. ${ }^{42,43}$ Furthermore, the usage of these IGHV and IGHD subgroups and of individual IGHJ segments were similar between controls and cosmonauts, thereby indicating that natural biases in subgroups and segment usages were not affected by a long-term space mission. We also noted that the peripheral blood IgM repertoire of control subjects was stable over an 8-month period, as previously shown for the bone marrow plasma cell immunoglobulin heavy chain repertoire ${ }^{44}$ and the adult peripheral blood IGHV repertoire. ${ }^{45}$

Our data revealed that the repertoire of unique VDJ associations in IgM transcripts of cosmonauts was different from that of controls already before launch. This difference is unlikely to be due to lifetime exposure to pathogens in different countries because one cosmonaut (COS4) originated from the same country as the controls and presented the same changes in the IgM repertoire as the other cosmonauts. Vaccination history is not either a likely explanation because both cosmonauts and control subjects received immunizations recommended for adults younger than 60 without disease and against Hepatitis A and B. The only difference was that cosmonauts were also immunized against Streptococcus pneumoniae/Pneumococcus and Neisseria meningitides/Meningococcus. Since recently, ISS astronauts are 
immunized against Varicella zoster virus (VZV). However, as an immunization against VZV was not recommended prior to December 2018, it can be expected that our subjects and all members of the control group did not receive the immunization against VZV. Our data demonstrated that the IgM repertoire of cosmonauts before flight was different from that of controls because it was much more reworked during B lymphopoiesis as more RAG-mediated secondary recombination of previously rearranged IgH gene segments occurred, as attested by the study of IGHV replacement signatures. This difference in repertoire might perhaps be due to the special regimes endured by cosmonauts to prepare them for spaceflight, as it was shown, for example, that training impacts humoral immunity. ${ }^{46-48}$

We then analyzed the effects of spaceflight on the $\operatorname{IgM}$ repertoire of the five cosmonauts. No obvious change was noted in COS1, 2 \& 3 when analyzing their repertoire as a whole. In the same way, another study showed that the antibody repertoire of mice flown to and housed on the ISS over a short period of time (21-22 days) was not affected. ${ }^{49}$ However, the analysis of associations involving the most expressed IGHV subgroups and the most expressed IGHJ segments revealed that some specific associations (IGHV3-48 with IGHJ5 and IGHV3-23 with IGHJ6) are altered in all the cosmonauts. Interestingly, IGHV3-48 and IGHV323 belong to the top 10 of the expressed IGHV segments and IGHJ6 is the second most frequently used IGHJ gene segment in the blood of healthy human adults. ${ }^{43}$ Thus, changes in the expression of these associations, if confirmed by future studies, likely have a meaning that will require investigations to determine their impacts on the immunocompetence of astronauts. Indeed, IGHV3-23 is regularly overrepresented in responses to many antigens. ${ }^{50}$ It was for example shown that IGHV3-23 is utilized with either IGHJ4 or IGHJ6 in the human antibody response to the Haemophilus influenzae type b (Hib) polysaccharide (PS) to encode an antibody combining site that is expressed in the majority of individuals and that comprises on average more than half the serum anti-Hib PS antibody population. ${ }^{51}$

The repertoire of VDJ associations in the other two cosmonauts, COS4 and COS5, was much more affected. Changes of CDR3 AA composition and of two out of the three signatures left by RAG in IgM transcripts, indicative of a modification of the $\mathrm{V}(\mathrm{D}) \mathrm{J}$ recombination genetic machinery, were much more pronounced in COS4 and COS5 than in COS1, 2 and 3 during the flight and recovery periods. We showed that these differences are not associated to inflight HSV-1/2 reactivation, an observation in line with a previous work investigating US astronauts. ${ }^{37}$ This observation suggests a lack of immunosuppression in spite of these changes to the $\operatorname{IgM}$ repertoire. Differences in $\operatorname{IgM}$ repertoire are unlikely due to immunization history as our cosmonauts were subjected to the same vaccination practice. These can neither be attributed to differences in extravehicular activities (EVAs) because COS4 did not perform an EVA, COS5 did perform three EVAs (5h 25m, 5h 38m, 7h 41m) without any reported issues, COS1 performed 1 EVA (6h 15m) without any reported abnormalities, COS2 performed 1 EVA $(5 \mathrm{~h} 51 \mathrm{~m})$ and COS3, who participated in the same mission as COS2, did not perform an EVA. Thus, EVAs were well-balanced in both groups (COS1, 2, 3 vs. COS4, 5). Furthermore, it is unlikely that these differences in repertoire resulted from mission differences, such as for example habitat microbial load or composition, because these five cosmonauts were 
involved in four different missions: COS1 took part in a mission occurring six months before that involving $\operatorname{COS} 2$ and 3; COS4 and COS5 took part in distinct missions separated by a 10-month interval. Furthermore, the most experienced cosmonaut (COS2), who participated in 3 previous missions, was not the most affected one. Such heterogeneity of response to the space exposome has already been noted in flown mice ${ }^{49}$ and in astronauts. Indeed, it was observed that $4 / 8$ crewmembers involved in a 6-month mission on the ISS shed at least one latent type of herpes virus and that this was associated with elevations in salivary cortisol, indicating stress-induced activation of the hypothalamic-pituitary adrenal (HPA) axis in these four subjects. ${ }^{52}$ Another study showed that $61 \%$ of astronauts from ISS missions shed one or more herpes viruses in saliva or urine samples. ${ }^{36}$ Thus, caveats should be strongly considered before averaging data because this process can hide individual responses. Furthermore, we observed that these modifications persisted during the whole mission and up to 30 days after landing, indicating that specificities of IgM binding sites are likely modified in these two subjects for a long period. Even if our sample size is small, it is tempting to put this observation in parallel with the study that showed that $46 \%$ of the astronauts who stayed 6 months on board the ISS encountered immunological changes. ${ }^{2}$ Additionally, differences in V(D)J recombination effector signatures observed in COS4 and COS5 suggest a modification of B lymphopoiesis in these two subjects, as observed in mice that spent 30 days onboard the BION-M1 satellite. ${ }^{23}$ Interestingly, these two subjects, as well as the BION-M1 mice, ${ }^{53}$ developed a stress response, and it was shown that exposure to glucocorticoids has a rapid effect on B lymphopoiesis because the administration of corticosterone induces a loss of 30-70\% in $24 \mathrm{~h}$ among murine early pro-B, pre-B and immature B cells. ${ }^{54}$ Thus, an individual's stress perception and response is a likely contributor to IgM repertoire changes, as previously shown for viral reactivation. ${ }^{36,52}$ In line with this, it was shown that exposure to hypergravity of $2 \mathrm{~g}$ for 21 days induces an increase in serum corticosterone and a modification of the $\mathrm{V}(\mathrm{D}) \mathrm{J}$ recombination machinery in murine pups. ${ }^{55}$

This study is limited by the inherently small sample size, the constraint of manned space missions, and the lack of an age- and sex-matched cosmonaut population remaining on Earth during the mission. Furthermore, we might have missed some unique rearrangements corresponding to rare B-cell clones as indicated by a study that did compare datasets generated with or without amplification ${ }^{56}$ but also because we performed these studies using a limited volume of whole blood due to sample sharing. However, we are confident in our results, as they fit perfectly with previous independent data that have repeatedly shown that between 46 and $52 \%$ of astronauts face immune changes during a space mission. ${ }^{1,2}$

In conclusion, this is the first study to show that the IgM repertoire of cosmonauts is different from that of control subjects prior to launch and that 2 out of the 5 analyzed cosmonauts present a modification of their IgM repertoire during a long-term mission on the ISS. Modifications noted in these two subjects persisted during the whole mission and up to 30 days after landing, correlated with changes of the V(D)J recombination machinery, coincided with a higher stress response, and likely affected the specificities of the IgM binding sites. These alterations of the antibody repertoire, combined with other immune alterations such as changes in cytokine production, inflammation, and immune cell population distributions and 
function, ${ }^{6,8,16,57-59}$ could increase the clinical impact on the immune system. Challenge-type experiments will need to be done to shed light on the consequences of these modifications of the antibody repertoire on immunocompetence and to estimate the effectiveness of inflight vaccination. Finally, the observation of individual responses indicates that personalized strategies should be considered to avoid inappropriate therapeutic treatments during future deep-space expeditions. Note that the results of this study go beyond astronaut health as they are also relevant for biomedical studies that deal with stress and unloading impacts (e.g. bed ridden) on host physiology and immunity.

\section{ACKNOWLEDGMENTS}

We thank all operators, scientists and administrators at Roscosmos (the Russian Space Agency), IBMP (the Institute for Biomedical Problems) and TsNIIMash in Russia, at DLR (the German Space Agency), at ESA (the European Space Agency) Moscow Office, at CNES (the French Space Agency) (Didier Chaput) as well as the team from the NASA Kennedy Space Centre and the Johnson Space Centre, who all made this project possible. We also express our thanks to Dr. Dominique Moser, Prof. Dr. Matthias Feuerecker, Marion Hörl and Katharina Biere for their support and to the former staff of the Thiel/Choukér laboratory, Prof. Dr. Manfred Thiel, PD Dr. Ines Kaufmann, Andrea Boltendahl, Camilla Ladinig and Iva Kumprey for their helping hands in realizing this project. We like to thank very much Dr. Bernd Johannes at DLR for support with the LME model and Dr. Andreas Osterman at the Max von Pettenkofer-Institute of the LMU, Munich, Germany, for the quantification of HSV-1/2 DNA. We also express our thanks to the ISS crew members who participated in this study with outstanding professionalism and enthusiasm.

This work was supported by CNES (grants DAR 4800000894, 4800000950 and 4800001008), the French Ministry of Higher Education and Research, the Université de Lorraine, the French State-Region Project Contract (CPER), the DLR on behalf of the Federal Ministry of Economics and Technology/Energy (grants BMWi 50WB0719, 50WB0919 and 50WB1319), ESA (ELIPS 3 and 4 programs), Roscosmos and the program of fundamental research (theme 65.1) of IBMP, Moscow.

In honor and memory of the cosmonaut, vice director of the IBMP and IMMUNO-co-PI Prof. Boris Morukov who passed away in 2015.

\section{CONFLICTS OF INTEREST STATEMENT}

The authors declare that they have no conflict of interest.

\section{AUTHOR CONTRIBUTIONS}

A. Choukér and J.-P. Frippiat designed research; S. Ghislin, J.-I. Buchheim, N. Ouzren-Zarhloul and S. Matzel performed research; J.-I. Buchheim, S. Ghislin and E. Albuisson performed statistical analyses; A. Vanet conceived the software required for this project; S. Ghislin and J.-I. Buchheim analyzed data; S. 
Ghislin, J.-I. Buchheim, A. Choukér and J.-P. Frippiat wrote the manuscript in conjunction with S. Ponomarev and M. Rykova.

\section{DATA AVAILABILITY}

Sequencing data from this publication have been deposited to the NCBI Sequence Read Archive (https://www.ncbi.nlm.nih.gov/sra) under accession number PRJNA627324.

\section{REFERENCES}

1. Kimzey SL. Hematology and immunology studies. In: Biomedical Results from Skylab. In: Johnson, RS., and Dietlein, L. F., eds; 1977:248-282.

2. Crucian B, Babiak-Vazquez A, Johnston S, Pierson DL, Ott CM, Sams C. Incidence of clinical symptoms during long-duration orbital spaceflight. Int J Gen Med. 2016;9:383-391.

3. Crucian BE, Choukèr A, Simpson RJ, et al. Immune system dysregulation during spaceflight: Potential countermeasures for deep space exploration missions. Front Immunol. 2018;9:1437.

4. Mehta SK, Crucian BE, Stowe RP, et al. Reactivation of latent viruses is associated with increased plasma cytokines in astronauts. Cytokine. 2013;61:205-209.

5. Guéguinou N, Huin-Schohn C, Bascove M, et al. Could spaceflight-associated immune system weakening preclude the expansion of human presence beyond earth's orbit? J Leukoc Biol. 2009;86:1027-1038.

6. Frippiat J-P, Crucian BE, de Quervain DJ-F, et al. Towards human exploration of space: The THESEUS review series on immunology research priorities. NPJ Microgravity. 2016;2:16040.

7. Makedonas G, Mehta S, Choukèr A, et al. Specific immunologic countermeasure protocol for deep-space exploration missions. Front Immunol. 2019;10:2407.

8. Buchheim J-I, Matzel S, Rykova M, et al. Stress related shift toward inflammaging in cosmonauts after long-duration space flight. Front Physiol. 2019;10:85.

9. Voss EW. Prolonged weightlessness and humoral immunity. Science. 1984;225:214-215.

10. Konstantinova IV, Rykova MP, Lesnyak AT, Antropova EA. Immune changes during long-duration missions. J Leukoc Biol. 1993;54:189-201. 
11. Stowe RP, Sams CF, Mehta SK, et al. Leukocyte subsets and neutrophil function after short-term spaceflight. J Leukoc Biol. 1999;65:179-186.

12. Rykova MP, Antropova EN, Larina IM, Morukov BV. Humoral and cellular immunity in cosmonauts after the ISS missions. Acta Astronautica. 2008;63:697-705.

13. Spielmann G, Agha N, Kunz H, et al. B cell homeostasis is maintained during long-duration spaceflight. J Appl Physiol. 2019;126:469-476.

14. Horie K, Sasanuma H, Kudo T, et al. Down-regulation of GATA1-dependent erythrocyte-related genes in the spleens of mice exposed to a space travel. Sci Rep. 2019;9:7654.

15. Boxio R, Dournon C, Frippiat J-P. Effects of a long-term spaceflight on immunoglobulin heavy chains of the urodele amphibian Pleurodeles waltl. J Appl Physiol. 2005;98:905-910.

16. Bascove M, Huin-Schohn C, Guéguinou N, Tschirhart E, Frippiat J-P. Spaceflight-associated changes in immunoglobulin VH gene expression in the amphibian Pleurodeles waltl. FASEB J. 2009;23:1607-1615.

17. Frippiat J-P. Contribution of the urodele amphibian Pleurodeles walt to the analysis of spaceflightassociated immune system deregulation. Mol Immunol. 2013;56:434-441.

18. Bascove M, Guéguinou N, Schaerlinger B, Gauquelin-Koch G, Frippiat J-P. Decrease in antibody somatic hypermutation frequency under extreme, extended spaceflight conditions. FASEB J. 2011;25:2947-2955.

19. Globus RK, Morey-Holton E. Hindlimb unloading: rodent analog for microgravity. J Appl Physiol. 2016;120:1196-1206.

20. Lescale C, Schenten V, Djeghloul D, et al. Hind limb unloading, a model of spaceflight conditions, leads to decreased B lymphopoiesis similar to aging. FASEB J. 2015;29:455-463.

21. Rettig TA, Bye BA, Nishiyama NC, et al. Effects of skeletal unloading on the antibody repertoire of tetanus toxoid and/or CpG treated C57BL/6J mice. PLoS ONE. 2019;14(1):e0210284.

22. Rettig TA, Nishiyama NC, Pecaut MJ, Chapes SK. Effects of skeletal unloading on the bone marrow antibody repertoire of tetanus toxoid and/or CpG treated C57BL/6J mice. Life Sci Space Res (Amst). 2019;22:16-28.

23. Tascher G, Gerbaix M, Maes P, et al. Analysis of femurs from mice embarked on board BION-M1 biosatellite reveals a decrease in immune cell development, including B cells, after $1 \mathrm{wk}$ of recovery on Earth. FASEB J. 2019;33:3772-3783. 
24. Schatz DG, Swanson PC. V(D)J recombination: mechanisms of initiation. Annu Rev Genet. 2011;45:167-202.

25. Ma Y, Pannicke U, Schwarz K, Lieber MR. Hairpin opening and overhang processing by an Artemis/DNA-dependent protein kinase complex in nonhomologous end joining and V(D)J recombination. Cell. 2002;108:781-794.

26. Huin-Schohn C, Guéguinou N, Schenten V, et al. Gravity changes during animal development affect IgM heavy-chain transcription and probably lymphopoiesis. FASEB J. 2013;27:333-341.

27. Pfaffl MW, Tichopad A, Prgomet C, Neuvians TP. Determination of stable housekeeping genes, differentially regulated target genes and sample integrity: BestKeeper--Excel-based tool using pair-wise correlations. Biotechnol Lett. 2004;26:509-515.

28. Choukèr A, Thiel M, Baranov V, et al. Simulated microgravity, psychic stress, and immune cells in men: observations during 120-day 6 degrees HDT. J Appl Physiol. 2001;90:1736-1743.

29. Alamyar E, Duroux P, Lefranc M-P, Giudicelli V. IMGT(®) tools for the nucleotide analysis of immunoglobulin (IG) and T cell receptor (TR) V-(D)-J repertoires, polymorphisms, and IG mutations: IMGT/V-QUEST and IMGT/HighV-QUEST for NGS. Methods Mol Biol. 2012;882:569-604.

30. Lange MD, Huang L, Yu Y, et al. Accumulation of VH replacement products in IgH genes derived from autoimmune diseases and anti-viral responses in human. Front Immunol. 2014;5:345.

31. Crooks GE, Hon G, Chandonia J-M, Brenner SE. WebLogo: a sequence logo generator. Genome Res. 2004;14:1188-1190.

32. Fonte C, Kaminski S, Vanet A, et al. Socioenvironmental stressors encountered during spaceflight partially affect the murine TCR- $\beta$ repertoire and increase its self-reactivity. FASEB J. 2019;33:896-908.

33. Rettig TA, Ward C, Pecaut MJ, Chapes SK. Validation of methods to assess the immunoglobulin gene repertoire in tissues obtained from mice on the International Space Station. Gravit Space Res. 2017;5:223.

34. Rettig TA, Ward C, Bye BA, Pecaut MJ, Chapes SK. Characterization of the naive murine antibody repertoire using unamplified high-throughput sequencing. PLOS ONE. 2018;13:e0190982.

35. Zhang Z, Burrows PD, Cooper MD. The molecular basis and biological significance of VH replacement. Immunol Rev. 2004;197:231-242. 
36. Rooney BV, Crucian BE, Pierson DL, Laudenslager ML, Mehta SK. Herpes virus reactivation in astronauts during spaceflight and its application on earth. Front Microbiol. 2019;10:16.

37. Mehta SK, Laudenslager ML, Stowe RP, et al. Latent virus reactivation in astronauts on the international space station. NPJ Microgravity. 2017;3:11.

38. Mehta SK, Laudenslager ML, Stowe RP, Crucian BE, Sams CF, Pierson DL. Multiple latent viruses reactivate in astronauts during Space Shuttle missions. Brain Behav Immun. 2014;41:210-217.

39. Mehta SK, Pierson DL. Reactivation of latent herpes viruses in cosmonauts during a soyuz taxi mission. Microgravity Sci Technol. 2007;19:215-218.

40. Mehta SK, Cohrs RJ, Forghani B, Zerbe G, Gilden DH, Pierson DL. Stress-induced subclinical reactivation of varicella zoster virus in astronauts. J Med Virol. 2004;72:174-179.

41. Kaminski DA, Wei C, Qian Y, Rosenberg AF, Sanz I. Advances in human B cell phenotypic profiling. Front Immunol. 2012;3:302.

42. Martin V, Wu Y-C, Kipling D, Dunn-Walters DK. Age-related aspects of human $\operatorname{IgM(+)~B~cell~}$ heterogeneity. Ann N Y Acad Sci. 2015;1362:153-163.

43. Hong B, Wu Y, Li W, et al. In-depth analysis of human neonatal and adult IgM antibody repertoires. Front Immunol. 2018;9:128.

44. Wu GC, Cheung N-KV, Georgiou G, Marcotte EM, Ippolito GC. Temporal stability and molecular persistence of the bone marrow plasma cell antibody repertoire. Nat Commun. 2016;7:13838.

45. Van Dijk-Härd I, Lundkvist I. Long-term kinetics of adult human antibody repertoires. Immunology. 2002;107:136-144.

46. Ledo A, Schub D, Ziller C, et al. Elite athletes on regular training show more pronounced induction of vaccine-specific T-cells and antibodies after tetravalent influenza vaccination than controls. Brain Behav Immun. 2020;83:135-145.

47. Campbell JP, Turner JE. Debunking the myth of exercise-induced immune suppression: Redefining the impact of exercise on immunological health across the lifespan. Front Immunol. 2018;9:648.

48. Shearer WT, Ochs HD, Lee B-N, et al. Immune responses in adult female volunteers during the bed-rest model of spaceflight: antibodies and cytokines. J Allergy Clin Immunol. 2009;123:900-905. 
49. Ward C, Rettig TA, Hlavacek S, Bye BA, Pecaut MJ, Chapes SK. Effects of spaceflight on the immunoglobulin repertoire of unimmunized C57BL/6 mice. Life Sci Space Res (Amst). 2018;16:63-75.

50. Mackworth-Young CG, Harmer IJ, Mageed RA. The role of antigen in the selection of the human V323 immunoglobulin heavy chain variable region gene. Clin Exp Immunol. 2003;134:420-425.

51. Liu L, Lucas AH. IGH V3-23*01 and its allele V3-23*03 differ in their capacity to form the canonical human antibody combining site specific for the capsular polysaccharide of Haemophilus influenzae type b. Immunogenetics. 2003;55:336-338.

52. Agha NH, Baker FL, Kunz HE, et al. Salivary antimicrobial proteins and stress biomarkers are elevated during a 6-month mission to the International Space Station. J Appl Physiol. 2020;128:264-275.

53. Novoselova EG, Lunin SM, Khrenov MO, et al. Changes in immune cell signalling, apoptosis and stress response functions in mice returned from the BION-M1 mission in space. Immunobiology. 2015;220:500-509.

54. Laakko T, Fraker P. Rapid changes in the lymphopoietic and granulopoietic compartments of the marrow caused by stress levels of corticosterone. Immunology. 2002;105:111-119.

55. Ghislin S, Ouzren-Zarhloul N, Kaminski S, Frippiat J-P. Hypergravity exposure during gestation modifies the TCR $\beta$ repertoire of newborn mice. Sci Rep. 2015;5:9318.

56. Rettig TA, Pecaut MJ, Chapes SK. A comparison of unamplified and massively multiplexed PCR amplification for murine antibody repertoire sequencing. FASEB Bioadv. 2019;1:6-17.

57. Crucian BE, Zwart SR, Mehta S, et al. Plasma cytokine concentrations indicate that in vivo hormonal regulation of immunity is altered during long-duration spaceflight. $J$ Interferon Cytokine Res. 2014;34:778-786.

58. Crucian B, Stowe RP, Mehta S, Quiriarte H, Pierson D, Sams C. Alterations in adaptive immunity persist during long-duration spaceflight. NPJ Microgravity. 2015;1:15013.

59. Guéguinou N, Jeandel J, Kaminski S, Baatout S, Ghislin S, Frippiat J-P. Modulation of Iberian ribbed newt complement component $\mathrm{C} 3$ by stressors similar to those encountered during a stay onboard the International Space Station. Int J Mol Sci. 2019;20:1579. 
Table 1. Primers sequences for RT-qPCR.

\begin{tabular}{|c|c|c|c|}
\hline \multirow[b]{2}{*}{ Target } & \multicolumn{2}{|c|}{ Sequence, 5'-3' } & \multirow[b]{2}{*}{$\begin{array}{c}\text { Annealing } \\
\text { temperature }\left({ }^{\circ} \mathrm{C}\right)\end{array}$} \\
\hline & Forward & Reverse & \\
\hline$B 2 M$ & ACACTGAATTCACCCCCACT & CGGCATCTTCAAACCTCCAT & 60 \\
\hline$U B C$ & GGGTCGCAGTTCTTGTTTGT & TCCAGCAAAGATCAGCCTCT & 61 \\
\hline $\operatorname{Ig} M$ & CAAGGACGTCATGCAGGGC & GCAGATGAGCTTGGACTTGC & 60 \\
\hline $\operatorname{Ig} D$ & TCCGTCACCTGCACACTGAA & AGCCACATCAGGAGGATGTT & 60 \\
\hline
\end{tabular}

Abbreviations: B2M, beta-2-microglobulin; RT-qPCR, reverse transcription quantitative polymerase chain reaction; $\mathrm{UBC}$, ubiquitin $\mathrm{C}$.

Table 2. Numbers of unique IgM heavy chain sequences. Means \pm confidence intervals (CIs).

\begin{tabular}{lllllllll} 
& Controls & \multicolumn{3}{c}{ Cosmonauts } \\
& T0 & T+8m & L-25 & F+64 & F+129 & R+1 & R+7 & R+30 \\
\hline Mean & 46173 & 53409 & 36932 & 54521 & 36549 & 34815 & 35374 & 35750 \\
$( \pm 95 \% \mathrm{CI})$ & $( \pm 21594)$ & $( \pm 35002)$ & $( \pm 16140)$ & $( \pm 34342)$ & $( \pm 62407)$ & $( \pm 22736)$ & $( \pm 17602)$ & $( \pm 37632)$ \\
\hline
\end{tabular}

The normality of the distribution was determined using the Shapiro-Wilk normality test, and homogeneity of variance was confirmed using the Levene test. As the normality of the distribution and homogeneity of variance were validated, one-way ANOVA was performed. No significant differences among these numbers of unique sequences were detected; consequently, no post hoc test was performed. 
Table 3. Functional IGHV gene segments detected in unique sequences of cosmonauts and controls analyzed in this study. + indicates that this segment was detected.

\begin{tabular}{|c|c|c|c|c|c|c|c|c|c|c|}
\hline Subgroup & $\begin{array}{l}\text { Gene } \\
\text { segment }\end{array}$ & COS1 & $\cos 2$ & COS3 & COS4 & COS5 & C1 & $\mathrm{C2}$ & $\mathrm{C3}$ & $\mathrm{C4}$ \\
\hline \multirow[t]{9}{*}{ IGHV1 } & IGHV1-2 & + & + & + & + & + & + & + & + & + \\
\hline & IGHV1-3 & + & + & + & + & + & + & + & + & + \\
\hline & IGHV1-8 & + & + & + & + & + & + & + & + & + \\
\hline & IGHV1-18 & + & + & + & + & + & + & + & + & + \\
\hline & IGHV1-24 & + & + & + & + & + & + & + & + & + \\
\hline & IGHV1-45 & + & + & + & + & + & + & + & + & + \\
\hline & IGHV1-46 & + & + & + & + & + & + & + & + & + \\
\hline & IGHV1-58 & + & + & + & + & + & + & + & + & + \\
\hline & IGHV1-69 & + & + & + & + & + & + & + & + & + \\
\hline \multirow[t]{2}{*}{ IGHV2 } & IGHV2-5 & + & + & + & + & + & + & + & + & + \\
\hline & IGHV2-70 & + & + & + & + & + & + & + & + & + \\
\hline \multirow[t]{19}{*}{ IGHV3 } & IGHV3-7 & + & + & + & + & + & + & + & + & + \\
\hline & IGHV3-9 & + & + & + & + & + & + & - & + & + \\
\hline & IGHV3-11 & + & + & + & + & + & + & + & + & + \\
\hline & IGHV3-13 & + & + & + & + & + & + & + & + & + \\
\hline & IGHV3-15 & + & + & + & + & + & + & + & + & + \\
\hline & IGHV3-20 & + & + & + & + & + & + & + & + & + \\
\hline & IGHV3-21 & + & + & + & + & + & + & + & + & + \\
\hline & IGHV3-23 & + & + & + & + & + & + & + & + & + \\
\hline & IGHV3-30 & + & + & + & + & + & + & + & + & + \\
\hline & IGHV3-33 & + & + & + & + & + & + & + & + & + \\
\hline & IGHV3-43 & + & + & + & + & + & + & + & + & + \\
\hline & IGHV3-48 & + & + & + & + & + & + & + & + & + \\
\hline & IGHV3-49 & + & + & + & + & + & + & + & + & + \\
\hline & IGHV3-53 & + & + & + & + & + & + & + & + & + \\
\hline & IGHV3-64 & + & + & + & + & + & + & + & + & + \\
\hline & IGHV3-66 & + & + & + & + & + & + & + & + & + \\
\hline & IGHV3-72 & + & + & + & + & + & + & + & + & + \\
\hline & IGHV3-73 & + & + & + & + & + & + & + & + & + \\
\hline & IGHV3-74 & + & + & + & + & + & + & + & + & + \\
\hline \multirow[t]{9}{*}{ IGHV4 } & IGHV4-4 & + & + & + & + & + & + & + & + & + \\
\hline & IGHV4-28 & + & + & + & + & + & + & + & + & + \\
\hline & IGHV4-30-1 & + & + & + & + & + & + & + & + & + \\
\hline & IGHV4-31 & + & + & + & + & + & + & + & + & + \\
\hline & IGHV4-34 & + & + & + & + & + & + & + & + & + \\
\hline & IGHV4-38-2 & + & + & + & + & + & + & + & + & + \\
\hline & IGHV4-39 & + & + & + & + & - & + & + & + & + \\
\hline & IGHV4-59 & + & + & + & + & + & + & + & + & + \\
\hline & IGHV4-61 & + & + & + & + & + & + & + & + & + \\
\hline \multirow[t]{2}{*}{ IGHV5 } & IGHV5-10-1 & + & + & + & + & + & + & + & + & + \\
\hline & IGHV5-51 & + & + & + & + & + & + & + & + & + \\
\hline IGHV6 & IGHV6-1 & + & + & + & + & + & + & + & + & + \\
\hline IGHV7 & IGHV7-4-1 & + & + & + & + & + & + & + & + & + \\
\hline Total & & 43 & 43 & 43 & 43 & 42 & 43 & 42 & 43 & 43 \\
\hline
\end{tabular}


Table 4 . Correlations between IgM repertoire variations observed during spaceflight and IGHD length or nucleotides trimmed off of the 5'-IGHJ ends.

\begin{tabular}{llll} 
& $\mathrm{F}+64$ & $\mathrm{R}+1$ & $\mathrm{R}+7$ \\
\hline IGHD length & $0.978(\mathrm{p}=0.011)^{*}$ & $0.867(\mathrm{p}=0.029)^{*}$ & $0.721(\mathrm{p}=0.084)^{\mathbf{t}}$ \\
Nt deleted at 5'-IGHJ ends & $0.965(\mathrm{p}=0.017)^{*}$ & $0.849(\mathrm{p}=0.035)^{*}$ & $0.764(\mathrm{p}=0.066)^{\mathbf{t}}$ \\
\hline
\end{tabular}

Before calculating Bravais-Pearson correlation coefficient values, distribution normality was assessed using the Shapiro-Wilk normality test. ${ }^{*} \mathrm{p} \leq 0.05,{ }^{\mathrm{t}} \mathrm{p}<0.10$, for unilateral $\mathrm{r}>0$ statistical test. 


\section{FIGURE LEGENDS}

Figure 1 B-cell percentages before and after a long-term mission on the ISS. Dot blots show individual Bcell percentages, as determined by flow cytometry, and mean values (red line) in cosmonauts $(\mathrm{n}=3-5)(\mathbf{A})$ and control subjects $(n=4)(B)$. B-cell percentages were found to significantly increase one day after returning from space $(\mathrm{R}+1)$ using the LME test and Bonferroni post hoc test. $* \mathrm{p}<0.05$ versus L-25. n.s. = non-significant.

Figure 2 Relative IgM and IgD mRNA expression in peripheral blood before, during and after a long-term mission on the ISS. Studies were performed before launch (L-25 (n=5)), inflight $(\mathrm{F}+64(\mathrm{n}=4)$ and F+129 $(\mathrm{n}$ =3) ) and after landing $(\mathrm{R}+1(\mathrm{n}=5), \mathrm{R}+7(\mathrm{n}=5)$ and $\mathrm{R}+30(\mathrm{n}=3))$. Dot blots show individual $\operatorname{IgM}$ and $\operatorname{IgD}$ mRNA relative expression and mean values (red line) in cosmonauts $(n=3-5)$ and controls $(n=4) . \operatorname{IgM}$ and IgD mRNAs were quantified by RT-qPCR, and their levels were normalized to two housekeeping transcripts. The relative value obtained with controls was set to 1 . No statistically significant differences were found using the LME test and Bonferroni post hoc test. n.s. = non-significant.

Figure 3 Spaceflight does not modify the percentages of functional and nonfunctional IgM mRNAs or the expression of IGHV and IGHD subgroups or IGHJ segments. IgM repertoires were analyzed, using highthroughput sequencing, at 6 time points: L-25 ( $n=5), F+64(n=4), F+129(n=3), R+1(n=5), R+7(n=5)$ and $\mathrm{R}+30(\mathrm{n}=3)$. Within these repertoires, we determined the frequency of functional and nonfunctional sequences (A) as well as the frequency of IGHV and IGHD subgroups and of IGHJ segments in functional sequences $(\mathbf{B})$. Data are shown as the means \pm confidence intervals. No statistically significant differences were found using the LME test and Bonferroni post hoc test. n.s. = non-significant.

Figure 4 The cosmonaut IgM repertoire is different from that of the controls. (A) Dot blots show individual dispersion indexes for unique VDJ associations in controls $(n=4)$ and cosmonauts $(n=5)$ at the beginning of the study (left panel) and 8 months later for controls or 7 days after landing for cosmonauts (right panel). (B) Dot blots show the individual frequency of IGHV replacement footprints at the same time points. Statistically significant differences were found using unpaired T-tests (A) and Mann-Whitney U tests (B). * $\mathrm{p} \leq 0.05 ; * * \mathrm{p} \leq 0.01$

Figure 5 Spaceflight affects the IgM repertoire of 2 out of 5 cosmonauts. The IgM repertoire was analyzed at 6 time points: $\mathrm{L}-25(\mathrm{n}=5), \mathrm{F}+64(\mathrm{n}=4), \mathrm{F}+129(\mathrm{n}=3), \mathrm{R}+1(\mathrm{n}=5), \mathrm{R}+7(\mathrm{n}=5)$ and $\mathrm{R}+30(\mathrm{n}=3)$. To evaluate the impact of spaceflight on this repertoire, for each cosmonaut, dispersion indexes determined for inflight and after-landing time points were compared to those determined before the flight (L-25). These index variations were compared to the variation of indexes determined using controls at T0 and T+8m (used 
as reference), and differences were expressed as heat maps in which variations within the confidence interval determined using control subjects are indicated in light gray, whereas those above or below control interval and considered to be different are in red and green, respectively. (A) Heat map indicating variations in dispersion indexes for all VDJ associations in the functional IgM repertoire of cosmonauts during and after the mission by comparison to before launch. $\mathrm{P}$ values calculated using the LME statistical model when comparing cosmonaut subgroups $\operatorname{COS} 1,2 \& 3$ vs. COS $4 \& 5$ are indicated under the heat map. ${ }^{\mathbf{t}}$ indicates a tendency $(\mathrm{p}<0.10)$. n.s. = non-significant. $(\mathbf{B})$ Heat map indicating variations in dispersion indexes for associations involving members of the most expressed IGHV subgroups (IGHV3 and IGHV4) and the most frequently used IGHJ segments (IGHJ4 to IGHJ6) in the functional IgM repertoire of cosmonauts during and after the mission by comparison to before launch. Black rectangles correspond to unavailable samples. Values on the right indicate differences by comparison to upper or lower borders of confidence intervals determined using controls.

Figure 6 Effect of spaceflight on CDR3. CDR3 sequences were analyzed at different time points: L-25 ( $\mathrm{n}=$ 5), $F+64(n=4), F+129(n=3), R+1(n=5), R+7(n=5)$ and $R+30(n=3)$. To evaluate the impact of spaceflight on cosmonaut CDR3s, we first determined the frequency of sequences according to the CDR3 length (A). Each line corresponds to the mean value for each time point in control subjects and cosmonauts. We also evaluated the effects of spaceflight on CDR3 amino acid composition (B). To address this question, we calculated dispersion indexes considering both the position and the frequency of each amino acid along CDR3 sequences of 9 to 24 AA. In these calculations, inflight and after-landing time points were compared to those determined before the flight (L-25). These index variations were compared to the variation of the indexes determined using controls at $\mathrm{T} 0$ and $\mathrm{T}+8 \mathrm{~m}$, and differences were expressed as heat maps in which variations within the confidence interval determined using control subjects are indicated in light gray, whereas those above or below the control interval and considered to be different are in red and green, respectively. Black rectangles correspond to unavailable samples. Values on the right indicate differences by comparison to upper or lower borders of confidence intervals determined using controls.

Figure 7 Effect of spaceflight on signatures left in IgM mRNAs by RAG1/2. To evaluate the impact on RAG1/2 activity, we studied the nucleotides (nt) deleted from the 3'-IGHV and 5'-IGHJ extremities as well as IGHD segment length. These signatures were analyzed at different time points: L-25 (n = 5), F+64 (n = 4), $F+129(n=3), R+1(n=5), R+7(n=5)$ and $R+30(n=3)$. Then, for each cosmonaut, dispersion indexes determined for each signature during and after the space mission were compared to those determined before the flight. These index variations were compared to the variation in the indexes determined using controls at $\mathrm{T} 0$ and $\mathrm{T}+8 \mathrm{~m}$, and differences were expressed as heat maps in which variations within the confidence interval determined using control subjects are indicated in light gray, whereas those above or below the control interval and considered to be different are in red and green, respectively. Black rectangles 
correspond to unavailable samples. Values on the right indicate differences obtained by comparing the data to the upper or lower borders of confidence intervals determined using controls. $\mathrm{P}$ values calculated using the LME statistical model when comparing cosmonaut subgroups COS1, $2 \& 3$ versus COS $4 \& 5$ are indicated under the heat map. ${ }^{\mathrm{t}}$ indicates a tendency $(\mathrm{p}<0.10) ;{ }^{*} \mathrm{p} \leq 0.05 ; * * \mathrm{p} \leq 0.01$; n.s. $=$ non-significant.

Figure 8 Stress markers during long-term spaceflight. (A) Dot plots show the CST scores calculated with the paper questionnaire in cosmonauts and controls in the morning and evening. No significant differences were found over time or between groups using the LME statistical model. (B) Cortisol levels were measured in the saliva of cosmonauts and controls in the morning and evening using an automated immunoassay system based on the principle of electrochemiluminescence. Statistically significant differences were found for evening cortisol levels in cosmonauts for F+64 $(n=4)$ and $R+1(n=5)$ versus L-25 using the LME model and Bonferroni post hoc test after Box-Cox transformation of data. Lower panels show cosmonaut subgroup analysis. COS1-3 (lower left panel) did not exhibit any significant changes in evening cortisol over time, whereas COS4 and COS5 (lower right panel) accounted for the changes observed at F+64 and R+1 versus L-25 in the whole group. ${ }^{*} \mathrm{p} \leq 0.05 ;{ }^{*} \mathrm{p} \leq 0.01 ; * * \mathrm{*} \leq 0.001$. n.s. $=$ non-significant. 

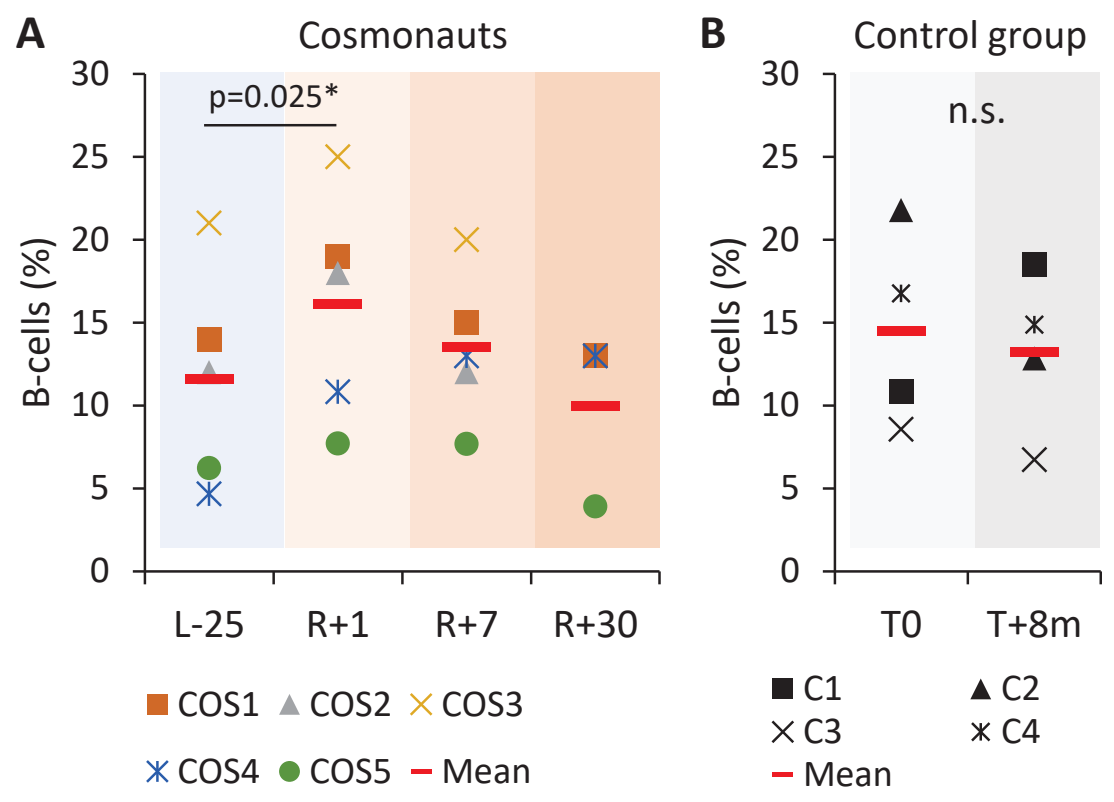
Figure 2

Figure 2

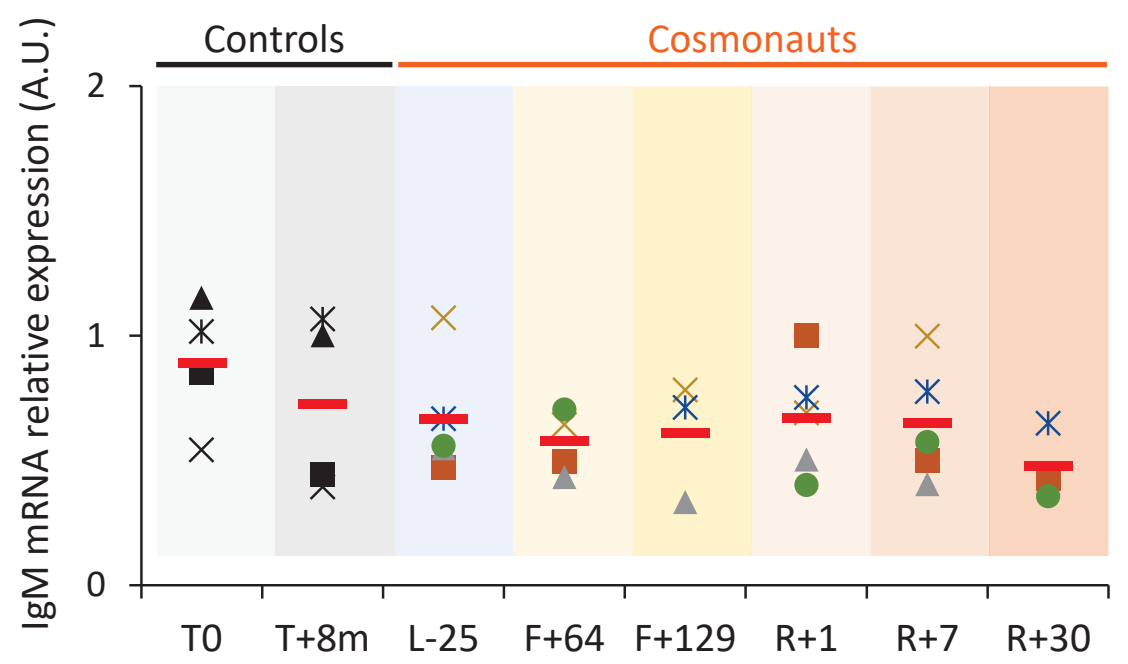

- C1

- C2

$\times \mathrm{C} 3$

* C4

n.s.

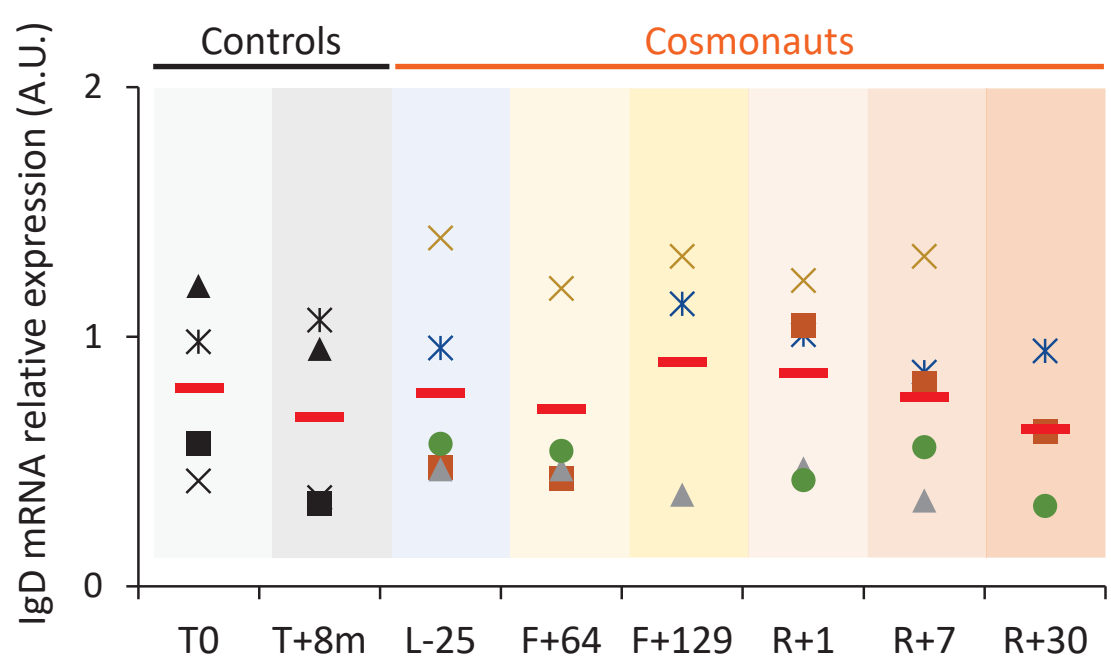

- cos1

- cos2

$\times \cos 3$

* cos4

- cos5

n.s. 
A

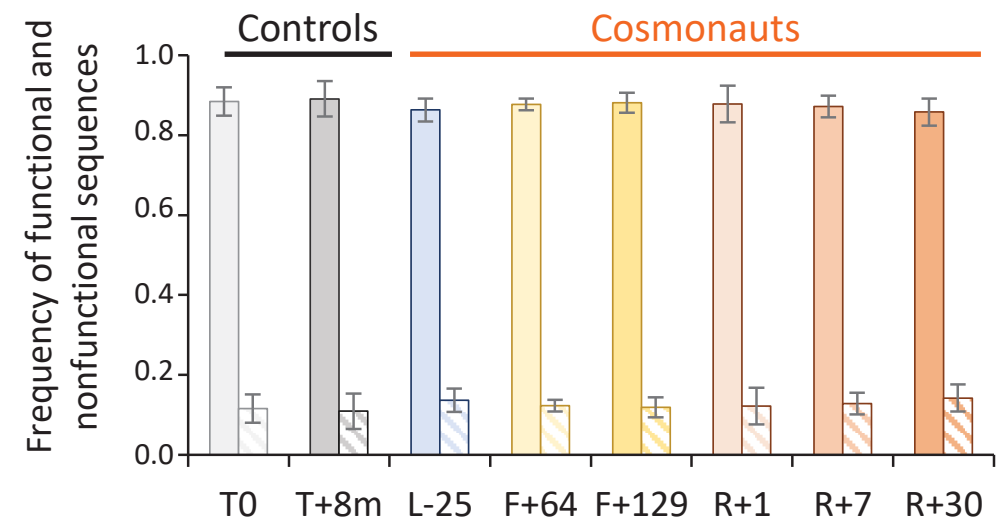

$\square$ Productive $\quad$ Unproductive

n.s.
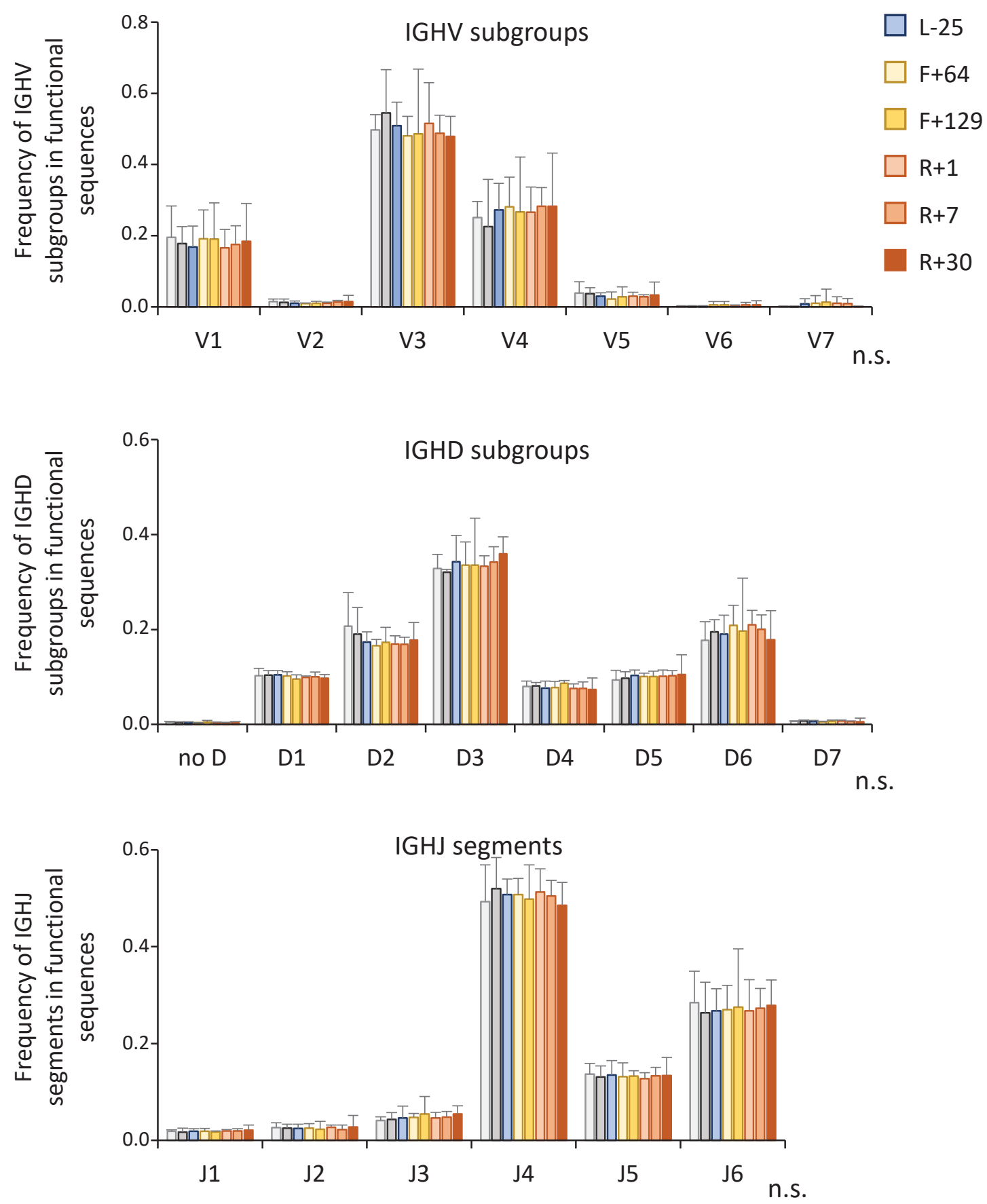
A
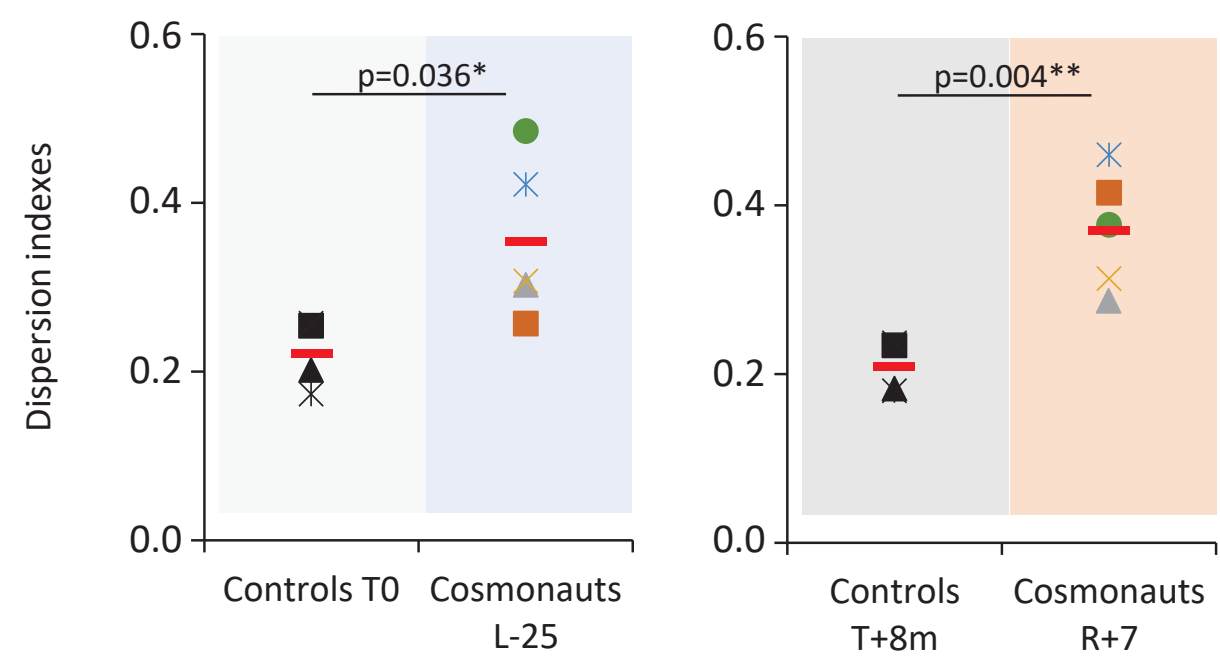

- C1

$\triangle \mathrm{C} 2$

$\times \mathrm{C} 3$

* C4

B
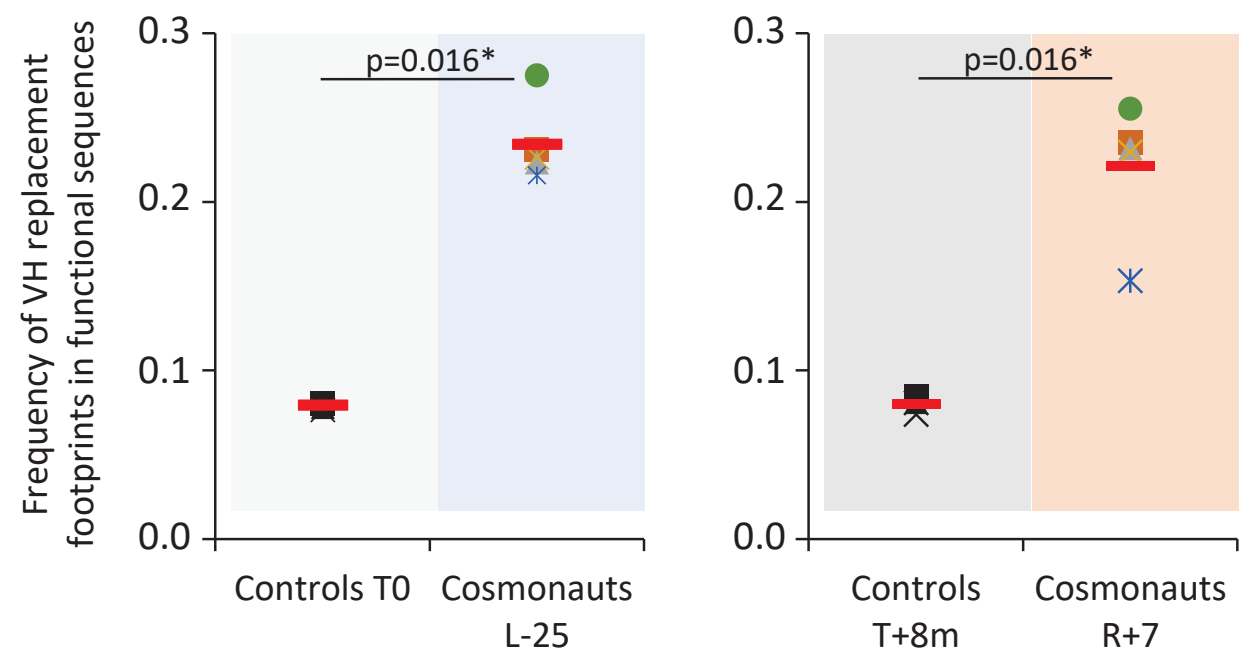

- cos1

- COS2

$\times \cos 3$

* cos4

- cos5

- Mean

L-25

$\mathrm{T}+8 \mathrm{~m}$

$\mathrm{R}+7$ 
Figure 5A

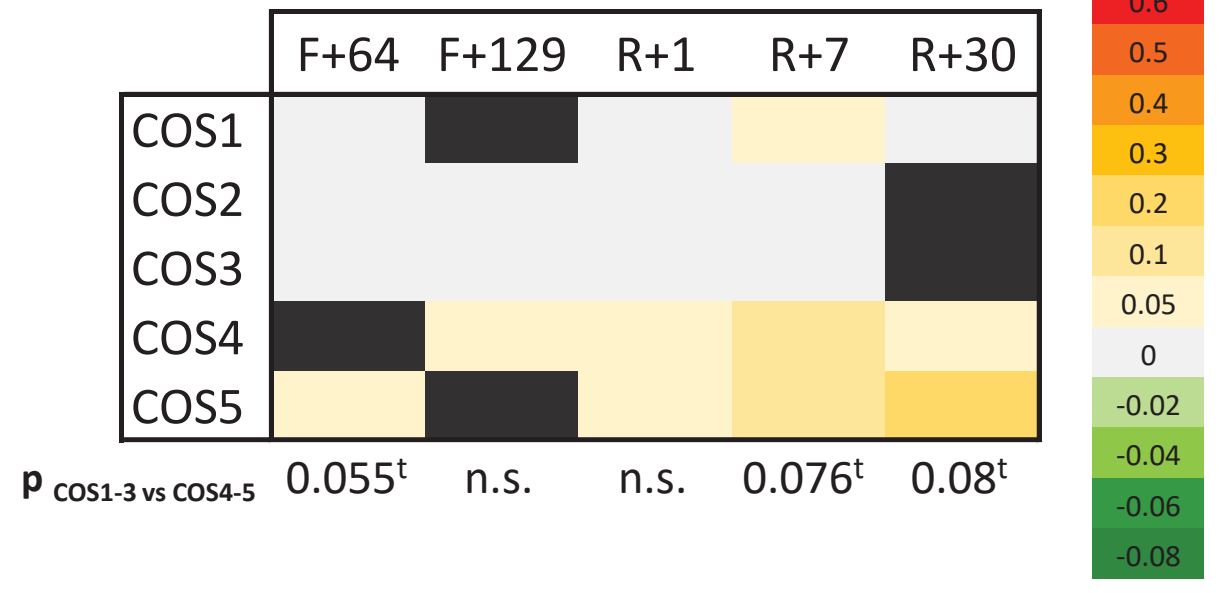


Figure 5B

Figure 5

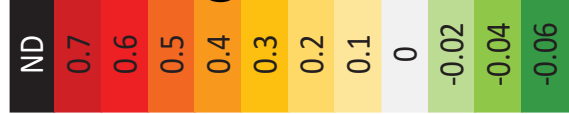
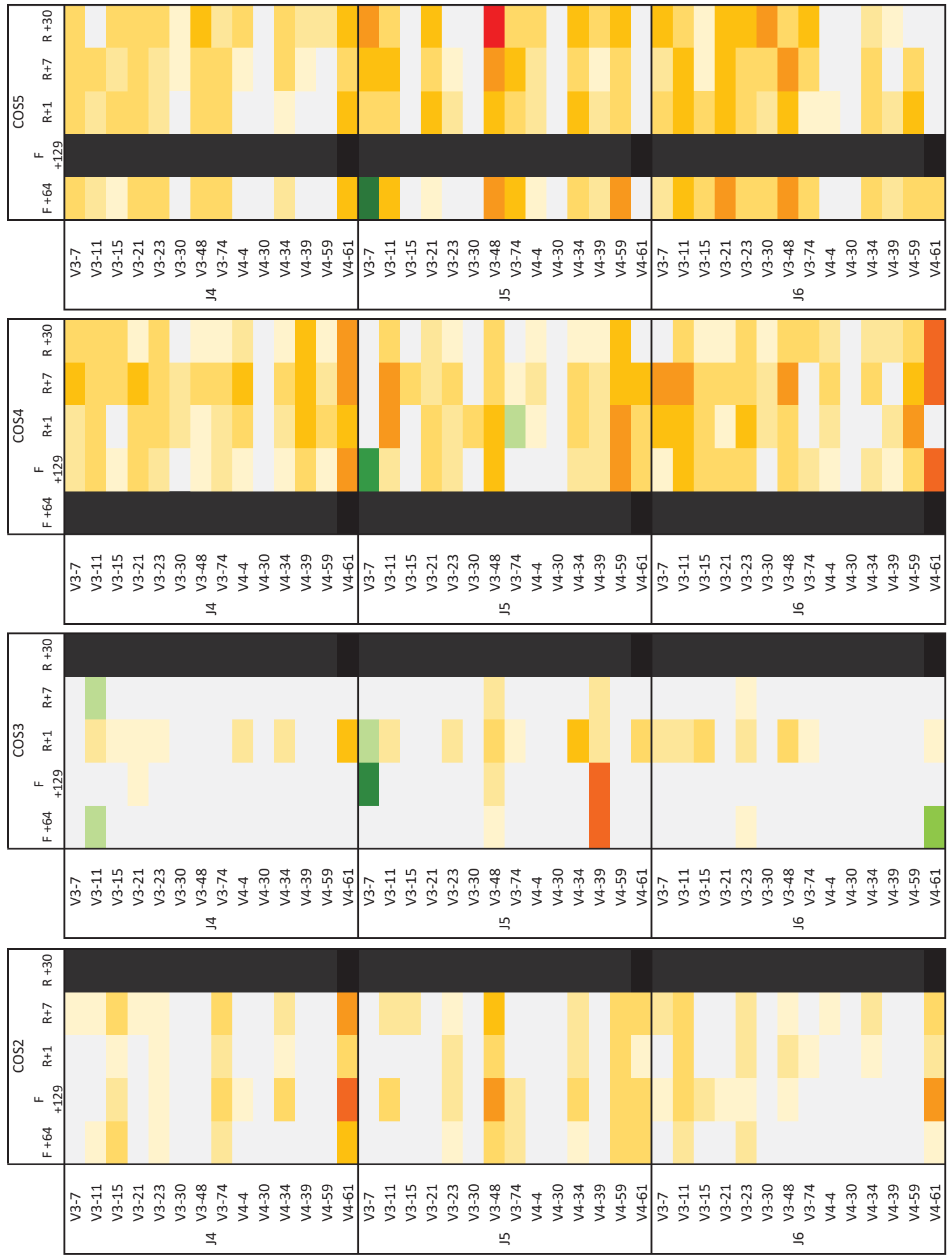

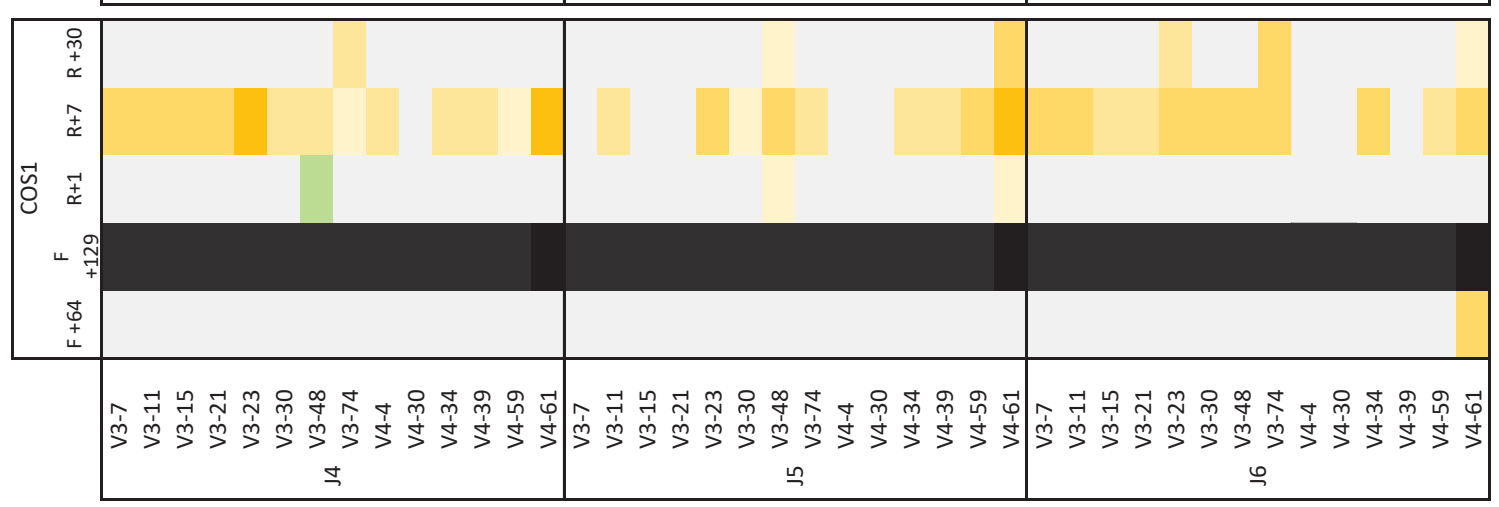


A

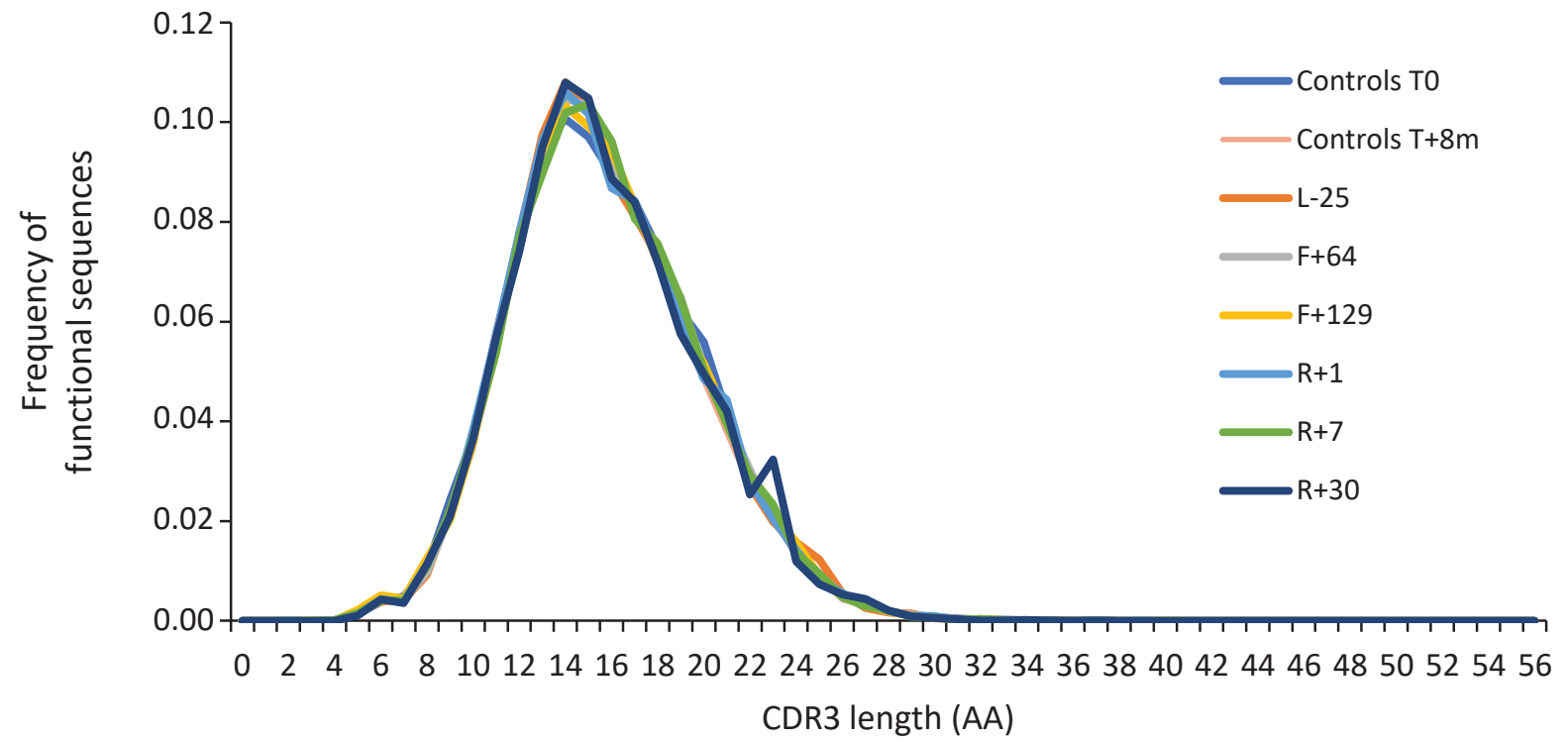

B
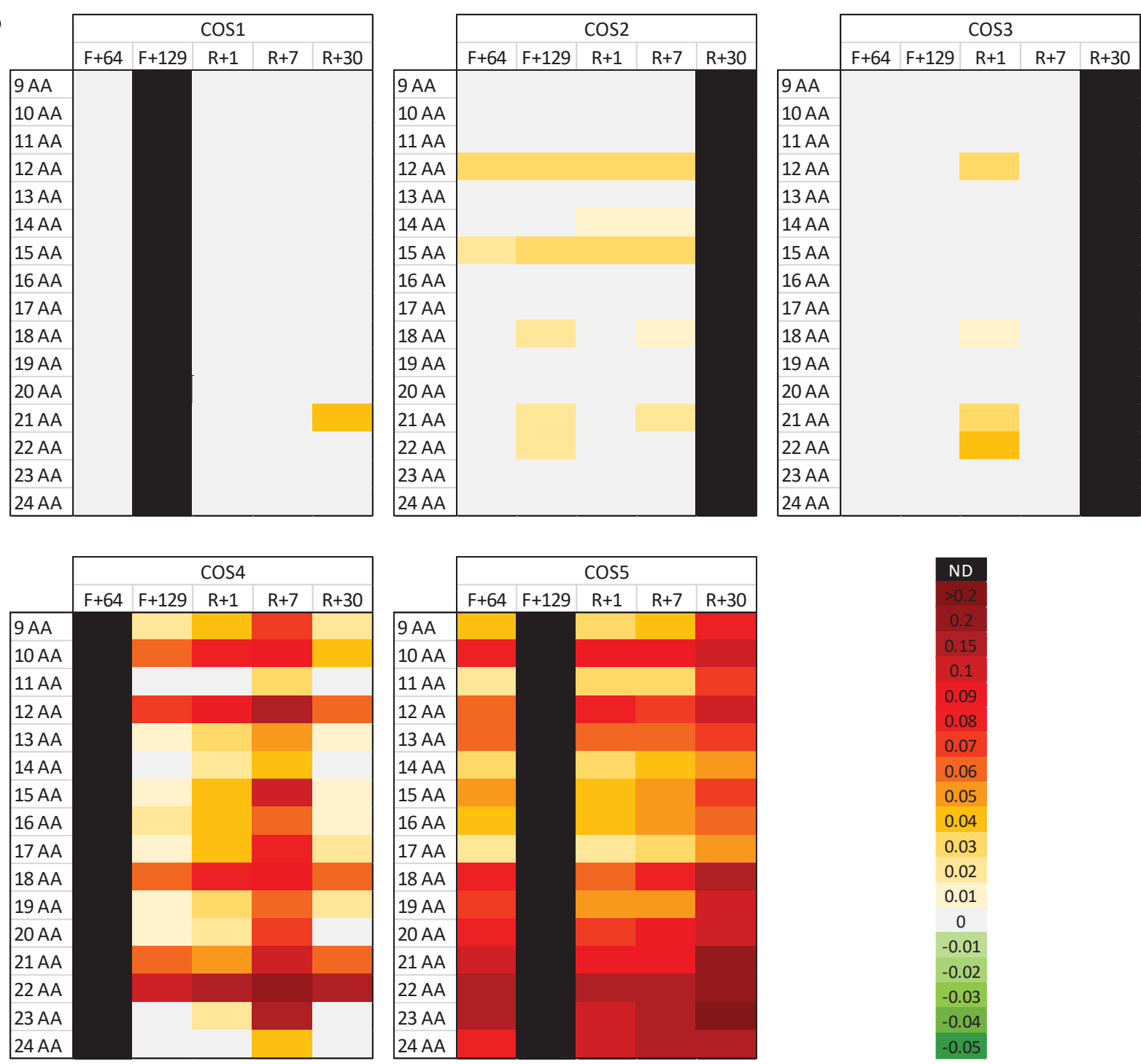


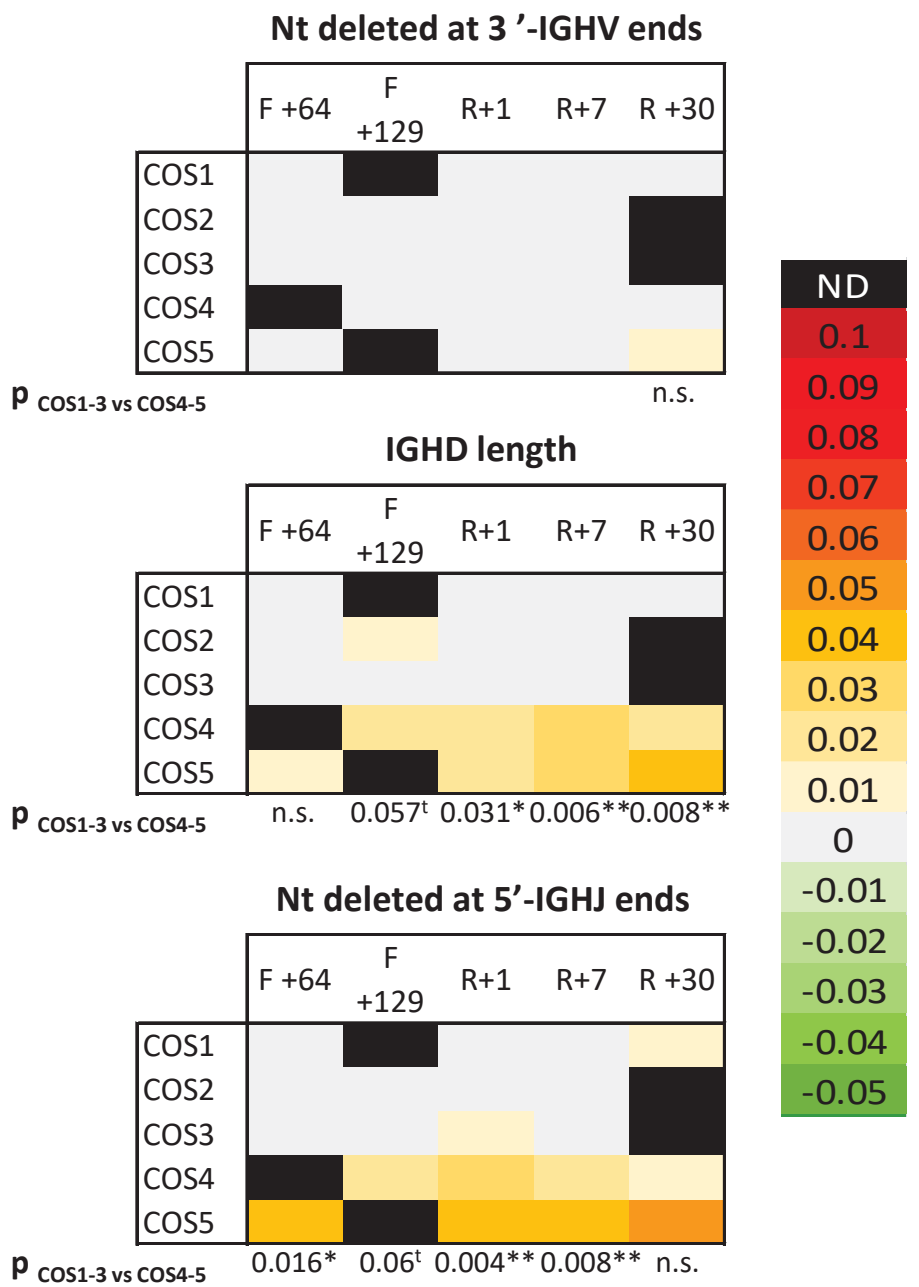


A

Stress test questionnaire

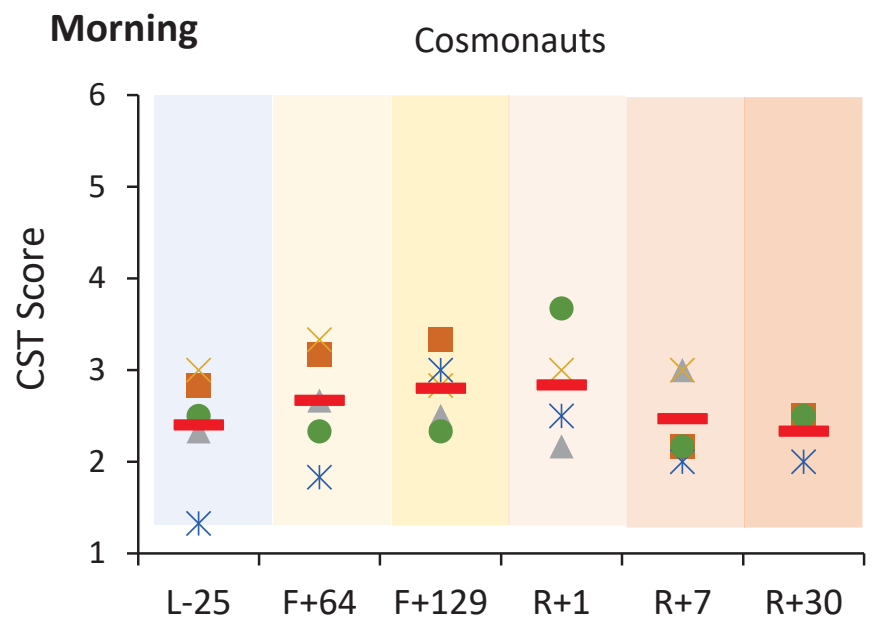

n.s.
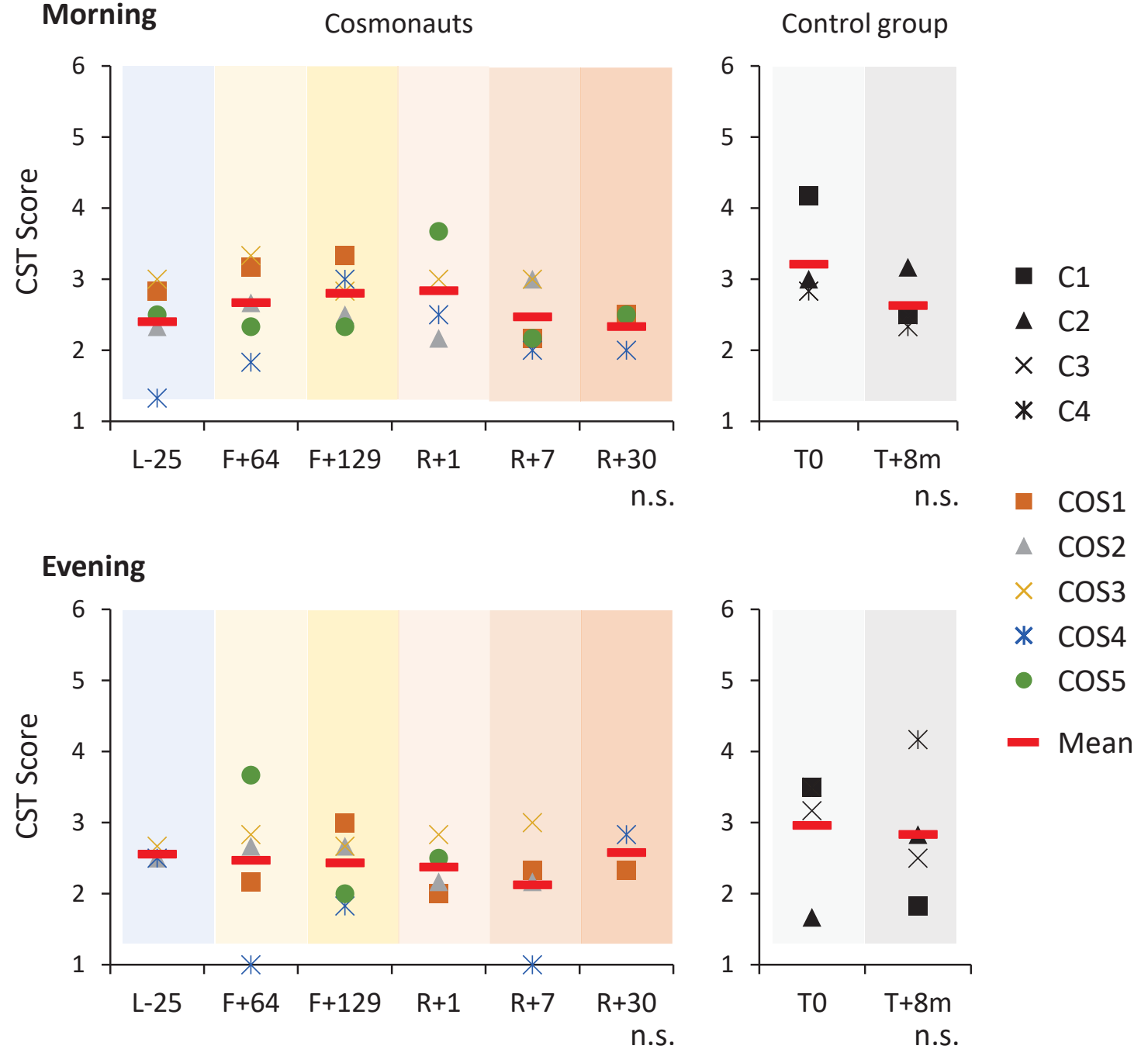
B

\section{Cortisol in saliva}

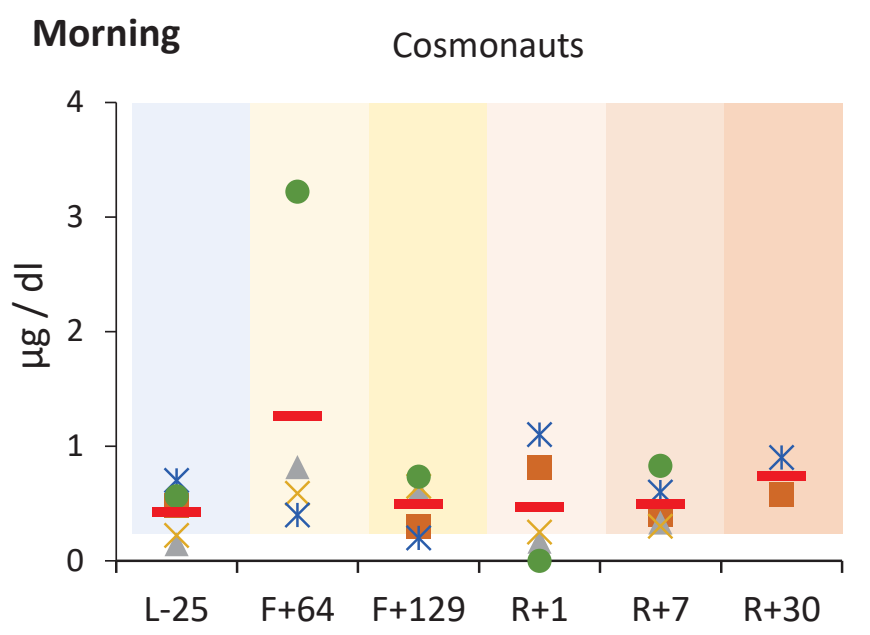

n.s.
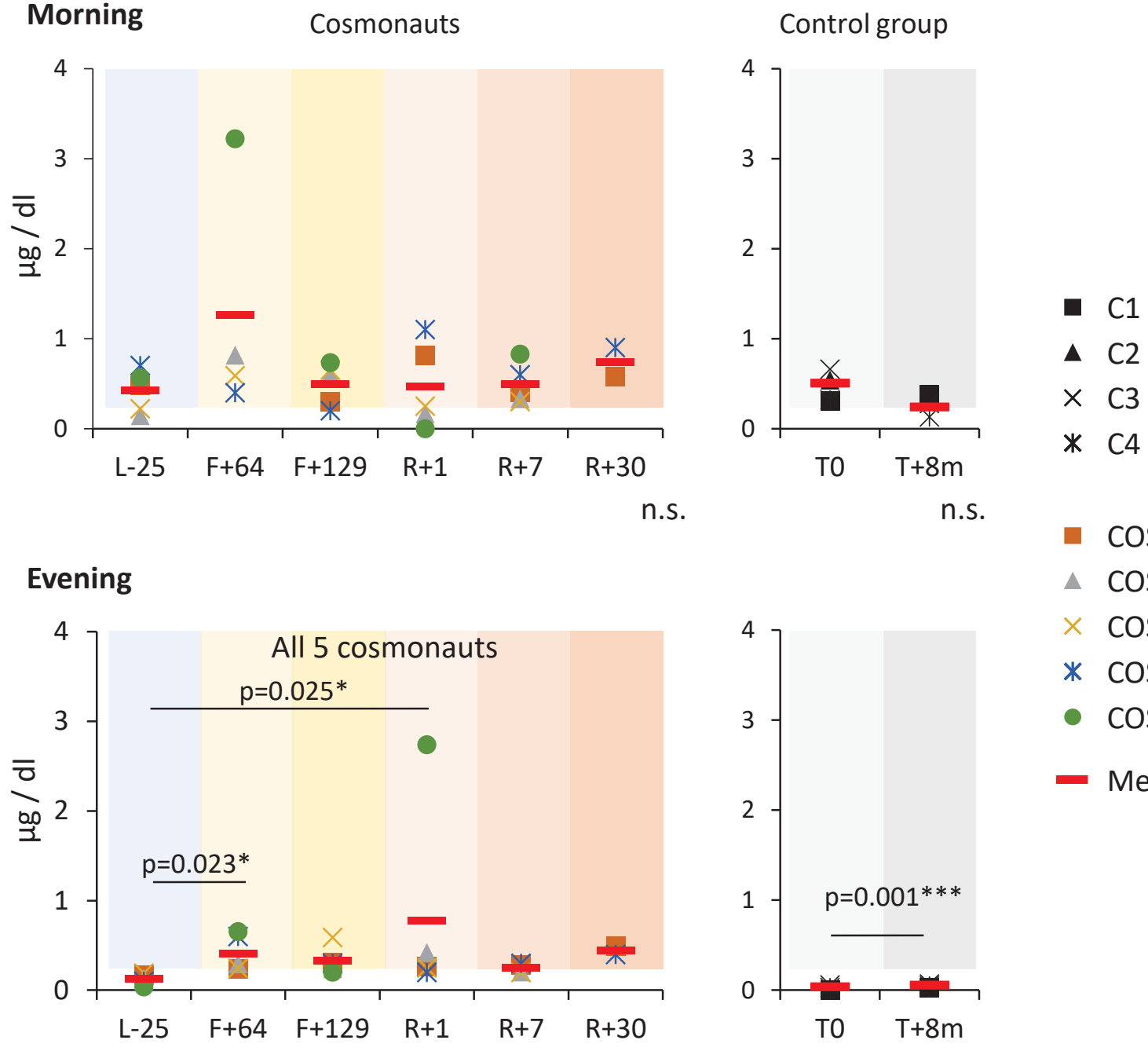

n.s.

- cos1

- cos2

$\times \cos 3$

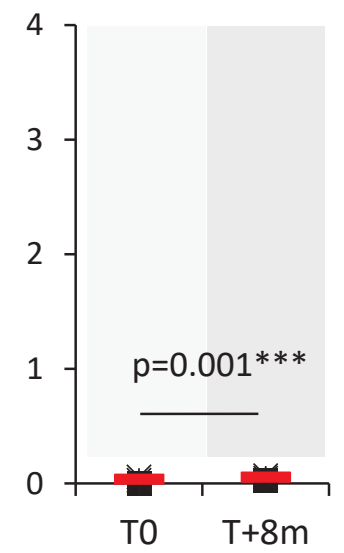

* cos4

- cos5

- Mean
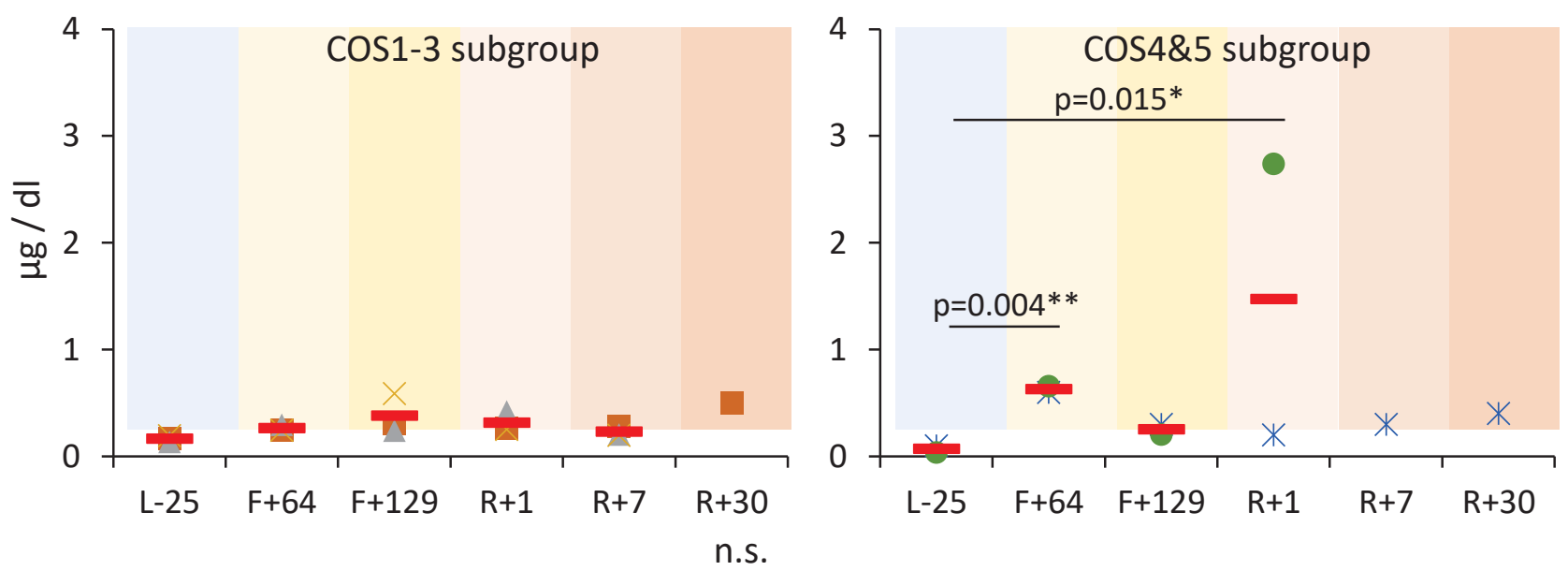